\title{
Exact solution of some quarter plane walks with interacting boundaries
}

\author{
N. R. Beaton A. L. Owczarek \\ School of Mathematics and Statistics \\ The University of Melbourne, \\ Victoria 3010, Australia \\ \{nrbeaton, owczarek\}@unimelb.edu. au
}

\author{
A. Rechnitzer \\ Department of Mathematics \\ The University of British Columbia, \\ Vancouver V6T 1Z2, British Columbia, Canada \\ andrewr@math.ubc.ca
}

Submitted: Jul 24, 2018; Accepted: Aug 9, 2019; Published: Sep 13, 2019

(c) The authors. Released under the CC BY-ND license (International 4.0).

\begin{abstract}
The set of random walks with different step sets (of short steps) in the quarter plane has provided a rich set of models that have profoundly different integrability properties. In particular, 23 of the 79 effectively different models can be shown to have generating functions that are algebraic or differentiably finite. Here we investigate how this integrability may change in those 23 models where in addition to length one also counts the number of sites of the walk touching either the horizontal and/or vertical boundaries of the quarter plane. This is equivalent to introducing interactions with those boundaries in a statistical mechanical context. We are able to solve for the generating function in a number of cases. For example, when counting the total number of boundary sites without differentiating whether they are horizontal or vertical, we can solve the generating function of a generalised Kreweras model. However, in many instances we are not able to solve as the kernel methodology seems to break down when including counts with the boundaries.
\end{abstract}

Mathematics Subject Classifications: 05A15

\section{Introduction}

The study of lattice random walks has a long history $[2,14,24,26]$. It is relatively straightforward to analyse random walks with different step sets when the walks are unrestricted. 
Once boundaries are introduced to restrict the region the problem can become far more difficult, and in some cases no solution has been found for what might seem simple regions. One restriction that has received much attention is to consider walks in the first quadrant of the plane with some subset of the 8 "short" steps $\{\mathrm{N}, \mathrm{E}, \mathrm{S}, \mathrm{W}, \mathrm{NE}, \mathrm{NW}, \mathrm{SE}, \mathrm{SW}\}$ see, amongst many others $[6,7,9,10,11,15,18,25]$. As usual from a combinatorial viewpoint the length generating function of all walk configurations is the mathematical object of interest.

In $[6,9,23]$ the 256 possible walk models are distilled into different equivalence classes. Of these classes, 79 (representing 138 distinct step sets) were identified as being non-trivial. Of these 79 , only 23 were determined to have algebraic or D-finite [22, 27] generating functions. In this paper we consider those 23 cases and extend the model by counting the number of times the random walk visits the horizontal and vertical boundaries. In the statistical physics and probability literatures this type of extension is described as adding an interaction on the boundary. Interaction problems are important as they often lead to so-called phase transitions [16] where the behaviour of the system changes markedly as the variable corresponding to surface visits is varied [28, 29]. Here our interest is in how counting the visits to the boundary changes the solvability of the problem and the analytic character of the generating function. In other words, how sensitive is that analytic character to the addition of boundary interactions?

\subsection{The model}

Consider a random walk on $\mathbb{Z}^{2}$ with steps $\mathcal{S}$ taken from the set $\{-1,0,1\}^{2} \backslash\{(0,0)\}$. The walks are restricted to lie in the non-negative quadrant — such random walks are known as quarter plane walks. A now classical problem is to enumerate the number of such random walks of length $n$ starting at the origin and then ending back at the origin, or ending anywhere in the quarter plane. In this paper we consider only those walks that start and end at the origin.

For a specific step set, let $q_{n}$ be the number of walks of length $n$ that start and end at the origin. We associate a generating function

$$
G(t)=\sum_{n \geqslant 0} q_{n} t^{n}
$$

In order to construct this generating function we need to form associated generating functions which count walks according to the coordinate of their end-point. To this end define

$$
Q(t ; x, y)=\sum_{n \geqslant 0} t^{n} \sum_{k, \ell \geqslant 0} q_{n, k, \ell} x^{k} y^{\ell}
$$

where $q_{n, k, \ell}$ is the number of walks of length $n$ ending at $(k, \ell)$. The nature of the generating functions $Q(t ; x, y)$, and in particular the specialisations $Q(t ; 0,0)$ and $Q(t ; 1,1)$, has been the focus of much work in this area.

We extend these enumeration problems by also counting the number of times walks visit either the horizontal or vertical boundaries. Let $q_{n, k, \ell, h, v}$ be the number of walks of 

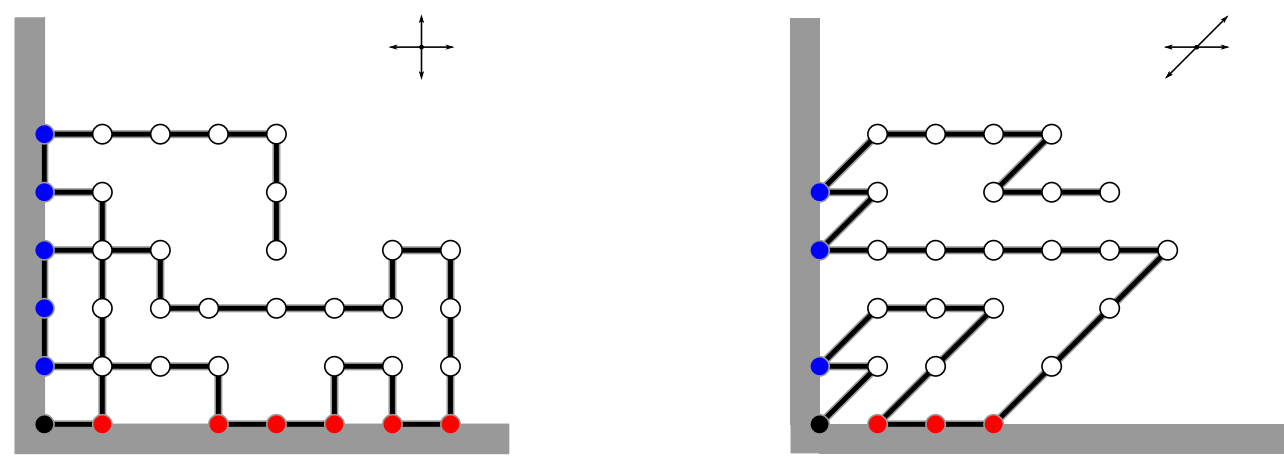

Figure 1: Two examples of random walks in the quarter plane with visits to either boundary wall indicated. The walk on the left may take N, S, E and W steps (see Model 1 below), while the walk on the right may take E, W, NE, and SW steps (see Model 23 below). The main focus of this paper is similar walks constrained to start and end at the origin.

length $n$ that end at $(k, \ell)$ which visit the horizontal boundary (ie. the line $y=0) h$ times, and visit the vertical boundary (ie. the line $x=0) v$ times. The associated generating function is

$$
Q(t ; x, y ; a, b) \equiv Q(x, y)=\sum_{n \geqslant 0} t^{n} \sum_{k, \ell, h, v \geqslant 0} q_{n, k, \ell, h, v} x^{k} y^{\ell} a^{h} b^{v} \equiv \sum_{n \geqslant 0} t^{n} Q_{n}(x, y) .
$$

A visit to the origin gains a weight $a b$ as the origin is considered as part of both the horizontal and vertical boundaries. We will, where it is clear, suppress the variables $t, a$ and $b$. We then redefine

$$
G(t ; a, b)=Q(t ; 0,0 ; a, b) .
$$

We examine four subcases: $G(t ; a, 1), G(t ; 1, b), G(t ; a, a)$ and $G(t ; a, b)$. These count (respectively) visits only to the horizontal boundary, visits only to the vertical boundary, visits to either boundary (not distinguishing between horizontal and vertical), and all visits to the boundaries (distinguishing between horizontal and vertical).

The nature of these generating functions for different values of $a, b$ is the focus of this paper.

\subsection{Existing results on weighted paths}

\subsubsection{Interacting directed polymers}

Part of the motivation for this paper stems from [28, 29], where models of interacting directed polymers were considered.

The subject of [28] was a model of two interacting polymers above an impenetrable wall. This comprised two paths of equal length in the half-space $y \geqslant 0$, each starting at $(0,0)$, and taking steps $(1,1)$ and $(1,-1)$ ("up" and "down" respectively). One path is designated as the "top" path, and is unable to cross below the other. Every vertex in the 


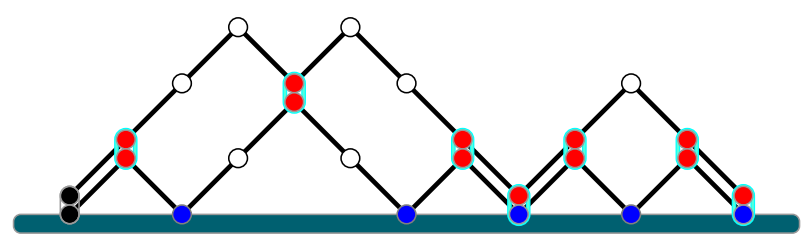

Figure 2: Two non-crossing directed paths of length 12 above a wall, with weight $a$ associated with visits to the boundary (blue circles) and weight $c$ associated with doubly-visited sites (red circles). This particular configuration has weight $a^{5} c^{7}$.

boundary $y=0$ visited by the bottom path (except the origin) accrues a weight $a$, and every vertex visited by both paths (except the origin) accrues a weight $c$. Vertices in the boundary visited by both paths accrue weight $a c$. See Figure 2.

The particular focus of [28] was pairs of paths which end together at a vertex in the surface, but it was necessary to also consider pairs which end at general heights. Let $f_{n, i, j, m_{a}, m_{c}}$ be the number of pairs of length $n$, with the bottom path ending at $(n, i)$ and the top at $(n, i+2 j)$, with $m_{a}$ vertices in the surface visited by the bottom path and $m_{c}$ shared vertices. In [28] is defined the generating function

$$
F(r, s, a, c ; z) \equiv F(r, s)=\sum_{n, i, j, m_{a}, m_{c}} f_{n, i, j, m_{a}, m_{c}} z^{n} r^{i} s^{j} a^{m_{a}} c^{m_{c}}
$$

By considering all the ways in which a pair of paths of length $n$ can be extended to one of length $n+1$, a functional equation for $F$ is found. This is first solved for the cases $a=1$ and $c=1$ separately, and these are later combined to give the general solution to $F(0,0)$.

There is a simple correspondence between this model and an interacting quarter plane model. Each pair of paths ending at heights $i$ and $i+2 j$ maps to a single quarter plane path ending at $(i, j)$. Then,

- when the pair of paths both step up, the quarter plane path steps east;

- when the pair of paths both step down, the quarter plane path steps west;

- when the top path steps up and the bottom steps down, the quarter plane path steps north-west; and

- when the top path steps down and the bottom path steps up, the quarter plane path steps south-east.

Note that then because the bottom path cannot cross the $x$-axis and the two paths cannot cross each other, the corresponding single path is exactly restricted to the quarter plane. Moreover, vertices in the boundary visited by the bottom path correspond to visits to the $y$-axis of the single path, and shared vertices correspond to visits to the $x$-axis. We thus see that, with $Q(t ; x, y ; a, b)$ defined as per $(3)$,

$$
F(r, s, a, c ; z)=Q(z ; r, s ; c, a),
$$


where the allowed step set for the quarter plane model is $\{(1,0),(-1,0),(-1,1),(1,-1)\}$ (Model 22 in Table 6).

The model studied in [29] is similar - instead of two non-crossing paths above a wall, the focus is three non-crossing paths (with no wall). By tracking the distance between the top/middle paths and the middle/bottom paths, a correspondence can be found with a single path in two dimensions; the non-crossing constraints ensure that the corresponding path lies in the quarter plane. However, this model is not exactly the same as one discussed in this paper, because there are two additional "neutral steps" (corresponding to the cases when all three paths step up or step down), when the quarter plane path does not move. Without these neutral steps one gets Model 18 in Table 3. The solution given in [29] is essentially the same as the one for Model 18 in Section 7.

\subsubsection{Other weighting schemes}

In [1], the authors find a connection between permutations sortable by two stacks in parallel and weighted quarter plane walks - specifically Model 1 walks which start and end at the origin. However, in this case the weights are not associated with visits to the boundaries - instead, a weight $a$ is associated with consecutive pairs of steps of the types north-west and east-south. A functional equation similar to (25) is derived for such walks.

In [19], the objects of investigation are quarter plane walks with multiplicities (integer weights) associated with each of the eight possible step directions. For simplicity they restrict the possible multiplicities to $0,1,2,3$ and 4 . This results in 30307 different nontrivial models, of which 1457 are D-finite and 79 are algebraic. With multiplicities the group structure can become more complicated, with $D_{10}$ appearing for three multiplicity sets; higher-order groups are likely to appear with greater multiplicities.

\subsection{The statistical physics interpretation}

We mentioned that the inclusion of weights $a, b$ on the boundaries corresponds to modelling interactions in a statistical physics context. Indeed, some quarter plane models correspond exactly to models of interacting directed polymers [28, 29]. Briefly, for a given model one can define the partition function

$$
P_{n}(a, b)=\left[t^{n}\right] Q(0,0),
$$

and set $a=e^{\alpha / k T}$ and $b=e^{\beta / k T}$, where $\alpha, \beta$ are the energies associated with visits to the walls, $T$ is absolute temperature and $k$ is Boltzmann's constant. Each partition function is then the normalisation for the Boltzmann distribution on walks of length $n$; for example, the probability of a walk of length $n$, starting and ending at the corner, with $i$ (resp. $j$ ) visits to the bottom (resp. left) wall is

$$
\frac{a^{i} b^{j}}{P_{n}(a, b)} .
$$

Observe that when $a$ is large (relative to $b$ ), walks with many visits to the bottom wall are favoured, while when $b$ is large, walks with many visits to the left wall are favoured. 


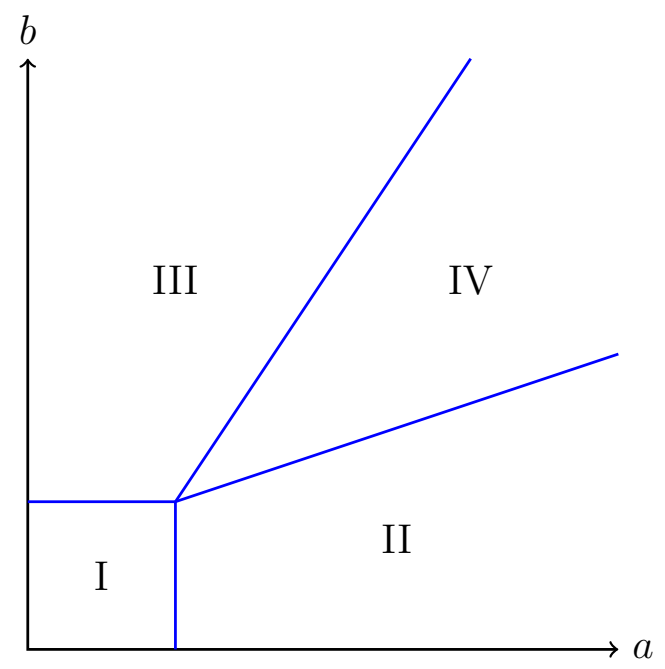

Figure 3: A possible schematic for the phase diagram of an interacting quarter plane walk model. In region I, the free energy $\kappa(a, b)$ is constant, so the densities of visits to both the bottom and left walls are zero. In region II (resp. III), $\kappa(a, b)$ depends only on $a$ (resp. $b$ ), so there is a positive density of visits to the bottom (resp. left) wall. In region $\mathrm{IV}, \kappa(a, b)$ depends on both $a$ and $b$, so both walls have a positive density of visits.

The (limiting) free energy of the model is

$$
\kappa(a, b)=\lim _{n \rightarrow \infty} \frac{1}{n} \log P_{n}(a, b) .
$$

The free energy describes the average behaviour of the walks as $n \rightarrow \infty$; for example, the average densities of visits to the bottom and left walls are

$$
\delta_{\mathrm{B}}(a, b)=a \frac{\partial}{\partial a} \kappa(a, b) \quad \text { and } \quad \delta_{\mathrm{L}}(a, b)=b \frac{\partial}{\partial b} \kappa(a, b) .
$$

The behaviour of $\kappa(a, b)$ in the $a-b$ plane, and in particular the regions where $\kappa$ depends on $a, b$, both, or neither, gives rise to the phases of the model. See Figure 3 for a possible schematic.

Note that $\left[t^{n}\right] Q(0,0)$ in $(7)$ could be replaced with $\left[t^{n}\right] Q(1,1)$, and the corresponding free energy may or may not change as a result.

For the purposes of this paper we are only interested in the combinatorics of the models in question, and we will thus not discuss the physical or probabilistic characteristics any further. These properties will be considered in a future publication.

\subsection{Main results}

Recall that a formal series in $t$ is differentiably finite (D-finite) $[22,27]$ if it satisfies a linear differential equation with integer polynomial coefficients in $t$. D-finite functions have some useful properties - most importantly, their coefficients satisfy finite linear recurrences. They are closed under addition, convolution and Hadamard products. Algebraic functions 
(roots of polynomials) are D-finite, and if $f(t)$ is D-finite and $a(t)$ is algebraic, then the composition $f(a(t))$ is D-finite.

A superset of D-finite functions are differentiably algebraic (D-algebraic) functions. A function $g(t)$ is D-algebraic if $g$ and its derivatives (with respect to $t$ ) satisfy a non-trivial polynomial. The ratio of two D-finite functions is D-algebraic, but in general not D-finite.

Proving that a given function is (or is not) D-finite or D-algebraic can sometimes be a difficult task. If $g(t)$ is D-finite, then it satisfies a differential equation

$$
p_{k}(t) g^{(k)}(t)+\cdots+p_{1}(t) g^{\prime}(t)+p_{0}(t) g(t)=0
$$

for some finite $k$. Every singularity (point of non-analyticity) of $g$ must be a root of $p_{k}(t)$, and this points to two methods for demonstrating that a function is not D-finite: show that it has infinitely many singularities (so that $p_{k}$ cannot be a polynomial), or show that it has a singularity at a transcendental number (so that $p_{k}$ cannot be an integer polynomial). We are not aware of a similar method for D-algebraic functions.

Since the coefficients of algebraic and D-finite functions satisfy certain kinds of recurrences, there exist tools for analysing series and "guessing" the corresponding polynomial or differential equation. One of the most powerful is the Ore Algebra package [17] in the Sage computer algebra system [30].

As noted above we focus on the 23 classes of step sets where the generating function $Q(t ; 0,0)$ for the non-interacting models is D-finite or algebraic: these are described in the tables below. As will be seen in Section 2, to some extent the generating functions $Q(t ; x, y), Q(t ; x, 0)$ and $Q(t ; 0, y)$ must also be considered, as they are necessary ingredients in the functional equations. However, for brevity and clarity our primary focus is $Q(t ; 0,0)$, and we will in general not discuss the properties of these other functions.

Our main results can be summarised as saying that the solubility of 22 of the 23 classes changes with the introduction of boundary weights $a$ and $b$. We follow the numbering from [9] and then separate the different walk classes according to the size of the symmetry group associated with their step set.

Each of Tables 1-6 contains information on the nature of the solution for various values of the boundary weights.

- Alg - the solution is algebraic (and not rational). If a checkmark $\checkmark$ is present, then this has been proved. If a cross $\boldsymbol{X}$ is present, then this has been guessed on the basis of analysis of series data using the Ore Algebra package.

- DF - the solution is D-finite. Again this has been proved where a $\boldsymbol{\checkmark}$ is present and guessed where there is an $\boldsymbol{X}$. In all the proven cases the solution is not algebraic. In the guessed cases we know that the solution cannot be algebraic if the solution at $(a, b)=1$ is not algebraic. This leaves Models 21 and 23; we have guessed D-finite solutions for some parameter ranges, but we have not been able to find algebraic solutions.

- DAlg — the solution is D-algebraic. We use a $\boldsymbol{\checkmark}$ to denote that the solution can be written as the ratio of two (known) D-finite functions and hence is D-algebraic. We have not been able to guess D-algebraic solutions to any of the models. 
- ? - we have not been able to solve or guess any algebraic, D-finite or D-algebraic solution.

In addition we give information about the so called group of the walk [11]. This is the group of symmetries satisfied by the kernel - see equation (16). We state the order of the group and the two generating involutions. We have used the notation $\bar{x} \equiv x^{-1}$ and $\bar{y} \equiv y^{-1}$.

\subsubsection{Vertically and horizontally symmetric $-D_{2}$ groups}

The 4 walk models in Table 1 possess both vertical and horizontal symmetry; the group of symmetries associated with their step set is equivalent to $D_{2}$. In all cases we are able to find the generating function for all $(a, b)$ values.

Theorem 1. For general values of $a$ and $b$, the generating function $G(t ; a, b)$ for Models 1 and 4 can be written as the ratio of two D-finite functions. This simplifies when either $a$ or $b$ is one, and $G(t ; a, 1)$ and $G(t ; 1, b)$ are D-finite. On the other hand, for Models 2 and $3, G(t ; a, b)$ is D-finite for all $a, b$.

Proof. See Sections 3 and 4.

\begin{tabular}{|c|c|c|c|c|c|c|c|}
\hline & Model & $(1,1)$ & $(a, a)$ & $(a, 1)$ & $(1, b)$ & $(a, b)$ & Group \\
\hline 1 & & $\mathrm{DF} \boldsymbol{}$ & DAlg $\boldsymbol{}$ & $\mathrm{DF} \boldsymbol{}$ & $\mathrm{DF} \boldsymbol{}$ & DAlg $\boldsymbol{}$ & 4: $(\bar{x}, y),(x, \bar{y})$ \\
\hline 2 & & $\mathrm{DF} \boldsymbol{}$ & $\mathrm{DF} \boldsymbol{}$ & $\mathrm{DF} \boldsymbol{}$ & $\mathrm{DF} \boldsymbol{}$ & $\mathrm{DF} \boldsymbol{}$ & $4:(\bar{x}, y),(x, \bar{y})$ \\
\hline 3 & & $\mathrm{DF} \boldsymbol{}$ & $\mathrm{DF} \boldsymbol{\nearrow}$ & $\mathrm{DF} \boldsymbol{}$ & $\mathrm{DF} \boldsymbol{}$ & $\mathrm{DF} \boldsymbol{\nearrow}$ & 4: $(\bar{x}, y),(x, \bar{y})$ \\
\hline 4 & $\stackrel{1}{\nearrow}$ & $\mathrm{DF} \boldsymbol{}$ & DAlg $\boldsymbol{}$ & $\mathrm{DF} \boldsymbol{\nearrow}$ & $\mathrm{DF} \boldsymbol{}$ & DAlg $\boldsymbol{}$ & 4: $(\bar{x}, y),(x, \bar{y})$ \\
\hline
\end{tabular}

Table 1: The step sets associated with these walk models are both vertically and horizontally symmetric. The results are summarised in Theorem 1 and the solutions are given in Sections 3 and 4 .

\subsubsection{Horizontally symmetric $-D_{2}$ groups}

The 12 walk models in Tables 2 and 3 possess horizontal symmetry - the step set is symmetric over the line $x=0$. The models in Table 2 have a net positive vertical drift (that is to say, there are more step directions which increase the $y$-coordinate than decrease it), while those in Table 3 have a net negative vertical drift. We were only able to 


\begin{tabular}{|c|c|c|c|c|c|c|c|}
\hline & Model & $(1,1)$ & $(a, a)$ & $(a, 1)$ & $(1, b)$ & $(a, b)$ & Group \\
\hline 5 & & $\mathrm{DF} \boldsymbol{\jmath}$ & $?$ & $\mathrm{DF} \boldsymbol{\jmath}$ & $?$ & $?$ & $4:(\bar{x}, y),\left(x, \frac{1}{x+\bar{x}} \bar{y}\right)$ \\
\hline 6 & & $\mathrm{DF} \boldsymbol{\jmath}$ & $?$ & $\mathrm{DF} \boldsymbol{\jmath}$ & $?$ & $?$ & 4: $(\bar{x}, y),\left(x, \frac{1}{x+\bar{x}} \bar{y}\right)$ \\
\hline 7 & & $\mathrm{DF} \boldsymbol{}$ & $?$ & $\mathrm{DF} \boldsymbol{}$ & $?$ & $?$ & 4: $(\bar{x}, y),\left(x, \frac{1}{x+1+\bar{x}} \bar{y}\right)$ \\
\hline 8 & & $\mathrm{DF} \boldsymbol{\nearrow}$ & $?$ & $\mathrm{DF} \boldsymbol{}$ & $?$ & $?$ & $4:(\bar{x}, y),\left(x, \frac{1}{x+1+\bar{x}} \bar{y}\right)$ \\
\hline 9 & & $\mathrm{DF} \boldsymbol{\nearrow}$ & $?$ & $\mathrm{DF} \boldsymbol{}$ & $?$ & $?$ & 4: $(\bar{x}, y),\left(x, \frac{x+\bar{x}}{x+1+\bar{x}} \bar{y}\right)$ \\
\hline 10 & & $\mathrm{DF} \boldsymbol{}$ & $?$ & $\mathrm{DF} \boldsymbol{}$ & $?$ & $?$ & $4:(\bar{x}, y),\left(x, \frac{x+\bar{x}}{x+1+\bar{x}} \bar{y}\right)$ \\
\hline
\end{tabular}

Table 2: Step sets that are symmetric over the line $x=0$ with a net positive vertical drift. We have only found solutions when $b=1$; the results are summarised in Theorem 2 and the solutions are given in Section 5 .

find solutions when $b=1$ - preserving the symmetry of the model. Indeed the solution method is essentially the same as for $a=b=1$ and the generating function remains D-finite.

When $b \neq 1$ we were not able to find or guess a solution for any of the models. We discuss this further in Section 5.

Theorem 2. The generating function $G(t ; a, 1)$ is D-finite for Models 5 to 16.

Proof. See Section 5.

\subsection{3 $\quad D_{3}$ groups}

Models 17 to 21 have diagonal symmetries rather than vertical or horizontal ones, and the corresponding group is isomorphic to $D_{3}$. The solutions to these models are varied.

Theorem 3. For general values of $a$ and $b$, the generating function $G(t ; a, b)$ for Model 17 can be written as the ratio of two D-finite functions. This simplifies when either a or $b$ is one, and $G(t ; a, 1)$ and $G(t ; 1, b)$ are D-finite. On the other hand, for Model 18, $G(t ; a, a)$ can be written as the ratio of two D-finite functions.

Proof. See Section 6 for a solution of Model 17, and Section 7 for a solution of Model 18. 


\begin{tabular}{|c|c|c|c|c|c|c|c|}
\hline & Model & $(1,1)$ & $(a, a)$ & $(a, 1)$ & $(1, b)$ & $(a, b)$ & Group \\
\hline 11 & & $\mathrm{DF} \checkmark$ & $?$ & $\mathrm{DF} \checkmark$ & $?$ & $?$ & $4:(\bar{x}, y),(x,(x+1+\bar{x}) \bar{y})$ \\
\hline 12 & & $\mathrm{DF} \boldsymbol{}$ & $?$ & $\mathrm{DF} \boldsymbol{\jmath}$ & $?$ & $?$ & $4:(\bar{x}, y),(x,(x+1+\bar{x}) \bar{y})$ \\
\hline 13 & & $\mathrm{DF} \checkmark$ & $?$ & $\mathrm{DF} \boldsymbol{\jmath}$ & $?$ & $?$ & $4:(\bar{x}, y),\left(x, \frac{x+1+\bar{x}}{x+\bar{x}} \bar{y}\right)$ \\
\hline 14 & & $\mathrm{DF} \boldsymbol{}$ & $?$ & $\mathrm{DF} \boldsymbol{}$ & $?$ & $?$ & $4:(\bar{x}, y),\left(x, \frac{x+1+\bar{x}}{x+\bar{x}} \bar{y}\right)$ \\
\hline 15 & & $\mathrm{DF} \boldsymbol{}$ & $?$ & $\mathrm{DF} \boldsymbol{}$ & $?$ & $?$ & 4: $(\bar{x}, y),(x,(x+\bar{x}) \bar{y})$ \\
\hline 16 & & $\mathrm{DF} \boldsymbol{}$ & $?$ & $\mathrm{DF} \boldsymbol{}$ & $?$ & $?$ & $4:(\bar{x}, y),(x,(x+\bar{x}) \bar{y})$ \\
\hline
\end{tabular}

Table 3: Step sets that are symmetric over the line $x=0$ with a net negative vertical drift. We have only found solutions when $b=1$; the results are summarised in Theorem 2 and the solutions are given in Section 5 .

Model 18 is very similar to the model considered in [29]; the main difference being the presence of a weighted neutral step. The solution we give in Section 7 is essentially unchanged from that in [29].

Notice that for Model 18, we have been able to guess D-finite solutions when either $a$ or $b$ is one, but have not been able to prove these rigorously. Additionally, for general $a, b$ we have not been able to find or guess any solution. We note that Model 17 has a single diagonal symmetry along the line $y=-x$ which is not that of the quarter plane itself which is symmetric on the line $y=x$. On the other hand Model 18 has two diagonal symmetries in the line $y=-x$ and the quarter plane symmetry of $y=x$. How this observation might be related to our difficulty in proving anything about Model 18 when $a \neq b$ which breaks the $y=x$ symmetry is an intriguing question.

\begin{tabular}{|c|c|c|c|c|c|c|c|}
\hline & Model & $(1,1)$ & $(a, a)$ & $(a, 1)$ & $(1, b)$ & $(a, b)$ & Group \\
\hline 17 & $\longleftarrow$ & DF $\boldsymbol{}$ & DAlg $\boldsymbol{}$ & DF $\boldsymbol{}$ & DF $\boldsymbol{}$ & DAlg $\boldsymbol{}$ & $6:(\bar{x} y, y),(x, x \bar{y})$ \\
\hline 18 & $\searrow$ \\
\hline
\end{tabular}

Table 4: Step sets associated with the group generated by the involutions given in the final column that generate a group isomorphic to $D_{3}$. 
Models 19, 20 and 21 are the Kreweras, reverse Kreweras and double Kreweras models $[8,9,20,23]$, and have algebraic generating functions when $a=b=1$. The only other model that does so is Model 23, Gessel walks [5, 13, 18]. We note Models 19 and 20 have a single diagonal symmetry $y=x$; the same as the quarter plane. Once again we can solve these models when that symmetry is preserved by setting $a=b$ in the boundary weights.

Theorem 4. For Models 19 and 20, the generating function $G(t ; a, a)$ is algebraic.

Proof. Since Models 19 and 20 are reverses of each other, any walk in Model 19 that starts and ends at the origin, is also a walk in Model 20 simply traversed backwards. Hence $G(t ; a, b)$ is identical in the two models. See Section 8 for a derivation of $G(t ; a, a)$.

Unfortunately, we have not been been able to solve either Models 19 or 20 when the $x=$ $y$ symmetry is broken. Despite this we have observed (again guessing via the Ore Algebra package) that $G(t ; a, b)$ is algebraic for general $(a, b) ; G(t ; a, 1)$ and $G(t ; 1, b)$ appear to satisfy degree 6 equations, while $G(t ; a, b)$ appears to satisfy a degree 12 equation. In addition, see the end of Section 8 for a discussion of recent work [3, 4] on Models 19 and 20 .

For Model 21, which has the two diagonal symmetries, we have guessed D-finite solutions when either $a$ or $b$ is one, but we have not found algebraic solutions. We have been unable to find any solution when $a=b$ or at general $a, b$.

\begin{tabular}{|c|c|c|c|c|c|c|c|}
\hline & Model & $(1,1)$ & $(a, a)$ & $(a, 1)$ & $(1, b)$ & $(a, b)$ & Group \\
\hline 19 & . & $\operatorname{Alg} \boldsymbol{V}$ & $\operatorname{Alg} \boldsymbol{V}$ & $\operatorname{Alg} \boldsymbol{X}$ & $\operatorname{Alg} \boldsymbol{X}$ & $\operatorname{Alg} \boldsymbol{x}$ & $6:(\overline{x y}, y),(x, \overline{x y})$ \\
\hline 20 & & $\operatorname{Alg} \boldsymbol{V}$ & $\operatorname{Alg} \boldsymbol{J}$ & $\operatorname{Alg} \boldsymbol{X}$ & $\operatorname{Alg} \boldsymbol{X}$ & $\operatorname{Alg} \boldsymbol{X}$ & $6:(\overline{x y}, y),(x, \overline{x y})$ \\
\hline 21 & & $\operatorname{Alg} \checkmark$ & $?$ & $\mathrm{DF} \boldsymbol{x}$ & DF $x$ & $?$ & $6:(\overline{x y}, y),(x, \overline{x y})$ \\
\hline
\end{tabular}

Table 5: Step sets associated with the group generated by the involutions given in the final column that generate a group isomorphic to $D_{3}$.

\subsection{4 $\quad D_{4}$ groups}

The final two step sets are neither symmetric under a single horizontal, vertical or either diagonal reflection. However, they are symmetric when double reflections are considered and the group of the walk is isomorphic to $D_{4}$. Nevertheless the integrability properties of their generating function differ. For Model 22 we can solve for the generating function for all boundary conditions. 
Theorem 5. For Model 22 the generating function $G(t ; a, b)$ is a ratio of $D$-finite functions which simplifies to a D-finite function if $a=1$ or $b=1$.

Proof. Model 22 is isomorphic to the problem studied in [28].

\begin{tabular}{|c|c|c|c|c|c|c|c|}
\hline & Model & $(1,1)$ & $(a, a)$ & $(a, 1)$ & $(1, b)$ & $(a, b)$ & Group \\
\hline 22 & + & $\mathrm{DF} \boldsymbol{\jmath}$ & DAlg $\boldsymbol{}$ & $\mathrm{DF} \boldsymbol{\jmath}$ & $\mathrm{DF} \boldsymbol{\jmath}$ & DAlg $\boldsymbol{}$ & 8: $(\bar{x} y, y),\left(x, x^{2} \bar{y}\right)$ \\
\hline 23 & & $\operatorname{Alg} \mathfrak{V}$ & $?$ & $\operatorname{Alg} \boldsymbol{X}$ & $\mathrm{DF} \boldsymbol{x}$ & $?$ & 8: $(\overline{x y}, y),\left(x, \bar{x}^{2} \bar{y}\right)$ \\
\hline
\end{tabular}

Table 6: Step sets associated with the group generated by the involutions given in the final column that generate a group isomorphic to $D_{4}$.

Unfortunately for Model 23 we could not derive a solution for any boundary values other than the original case of $a=b=1$. However, our series analysis leads us to suggest that the solution stays algebraic if $b=1$ but may be D-finite when $a=1$.

The organisation of the remainder of this paper is as follows. In Section 2 we lay out the general functional equation which will be the starting point for all 23 models. In Sections 3 and 4 we solve Models $1-4$. All horizontally symmetric models (5-16) are then considered together in Section 5. Models 17-19 are the focus of Sections 6-8, respectively. A final discussion and some open questions are presented in Section 9.

\section{The general functional equation}

For a given model, let $\mathcal{S} \subseteq\{-1,0,1\}^{2} \backslash\{(0,0)\}$ be the set of allowable steps. The step set generator is

$$
S(x, y)=\sum_{(i, j) \in \mathcal{S}} x^{i} y^{j} .
$$

We write, following the notation from [9], that

$$
S(x, y)=A_{-1}(x) \bar{y}+A_{0}(x)+A_{1}(x) y=B_{-1}(y) \bar{x}+B_{0}(y)+B_{1}(y) x .
$$

In the absence of any boundary weights, a functional equation for $Q(t ; x, y ; a, b) \equiv$ $Q(x, y)$ can be obtained by considering how one obtains all walks by the addition of a single step onto a walk of any length. Away from the boundaries adding a step gives $t S(x, y) Q(x, y)$ and the zero length walk is counted by a 1 . One cannot step south across the horizontal boundary so one needs to remove $t \bar{y} A_{-1}(x) Q(x, 0)$. Similarly one cannot step west across the vertical boundary so one needs to subtract $t \bar{x} B_{-1}(y) Q(0, y)$. Finally note that one has subtracted twice the contribution of walks stepping south-east from the 
origin and so we must add them back once as $t \overline{x y} \epsilon Q(0,0)$, where $\epsilon=\left[x^{-1} y^{-1}\right]\{S(x, y)\}$ and so is 1 if $(-1,-1) \in \mathcal{S}$ and 0 otherwise. So we obtain

$$
Q(x, y)=1+t S(x, y) Q(x, y)-t \bar{y} A_{-1}(x) Q(x, 0)-t \bar{x} B_{-1}(y) Q(0, y)+t \overline{x y} \epsilon Q(0,0) .
$$

This is often written as

$$
x y K(x, y) Q(x, y)=x y-t x A_{-1}(x) Q(x, 0)-t y B_{-1}(y) Q(0, y)+t \epsilon Q(0,0),
$$

where $K(x, y)$ is the kernel and is given by

$$
K(x, y)=1-t S(x, y) .
$$

With boundary weights, things become more complicated. We must account for the following:

(1) when a walk steps along the $x$-axis, but not to $(0,0)$, it accrues weight $a$;

(2) when a walk steps south onto the $x$-axis, but not to $(0,0)$, it accrues weight $a$;

(3) when a walk steps along the $y$-axis, but not to $(0,0)$, it accrues weight $b$;

(4) when a walk steps west onto the $y$-axis, but not to $(0,0)$, it accrues weight $b$; and

(5) when a walk steps onto the vertex at $(0,0)$, it accrues weight $a b$.

We define a more general indicator function: $\epsilon_{i j}$ is 1 if $(i, j) \in \mathcal{S}$, and is 0 otherwise, where + stands for 1 and - stands for -1 . We address the five points above in turn.

(1) To (14), we add

$$
t(a-1) \epsilon_{+0} x Q(x, 0)+t(a-1) \epsilon_{-0} \bar{x}(Q(x, 0)-Q(0,0))-t(a-1) \epsilon_{-0}\left[x^{1}\right]\{Q(x, 0)\} .
$$

We note that there is a factor of $(a-1)$ rather than just $a$ since all walks have already been enumerated but without the new weight. Hence we subtract off the incorrectly weighted walks and then add them back with the correct new weight. The first term accounts for walks moving east along the horizontal boundary, while the second term accounts for walks moving west along the horizontal boundary. It is more complicated because of the conditions around the origin. The next four points follow by similar arguments.

(2) Then add

$$
\begin{aligned}
& t(a-1) \epsilon_{+-} x\left[y^{1}\right]\{Q(x, y)\}+t(a-1) \epsilon_{0-}\left[y^{1}\right]\{Q(x, y)-Q(0, y)\} \\
& +t(a-1) \epsilon_{--} \bar{x}\left[y^{1}\right]\{Q(x, y)-Q(0, y)\}-t(a-1) \epsilon_{--}\left[x^{1} y^{1}\right]\{Q(x, y)\} .
\end{aligned}
$$

(3) Next add

$$
t(b-1) \epsilon_{0+} y Q(0, y)+t(b-1) \epsilon_{0-} \bar{y}(Q(0, y)-Q(0,0))-t(b-1) \epsilon_{0-}\left[y^{1}\right]\{Q(0, y)\} .
$$


(4) Then

$$
\begin{aligned}
& t(b-1) \epsilon_{-+} y\left[x^{1}\right] Q(x, y)+t(b-1) \epsilon_{-0}\left[x^{1}\right]\{Q(x, y)-Q(x, 0)\} \\
& \quad+t(b-1) \epsilon_{--} \bar{y}\left[x^{1}\right]\{Q(x, y)-Q(x, 0)\}-t(b-1) \epsilon_{--}\left[x^{1} y^{1}\right]\{Q(x, y)\} .
\end{aligned}
$$

(5) Finally, add

$$
\begin{aligned}
t(a b-1) \epsilon_{-0}\left[x^{1}\right]\{Q(x, 0)\}+t(a b-1) \epsilon_{0-}\left[y^{1}\right]\{ & Q(0, y)\} \\
& +t(a b-1) \epsilon_{--}\left[x^{1} y^{1}\right]\{Q(x, y)\} .
\end{aligned}
$$

Adding all these contributions gives an equation with 9 unknown terms. While this is very unwieldy to write down (so we won't), it is relatively simple to manipulate using computer algebra. Fortunately, 5 of these unknowns can be eliminated and the equation simplifies considerably, by using some additional relations. These relations are effectively obtained by extracting the coefficient of $x^{0}, y^{0}$ or $x^{0} y^{0}$, or equivalently by considering those walks that either end on a boundary or at the origin.

Firstly, a walk ending on the $x$-axis was previously either on the $x$-axis or immediately above it (or is the empty walk), so

$$
\begin{gathered}
Q(x, 0)=1+\operatorname{ta\epsilon }_{+0} x Q(x, 0)+\operatorname{ta\epsilon }_{-0} \bar{x}(Q(x, 0)-Q(0,0))+\operatorname{ta}(b-1) \epsilon_{-0}\left[x^{1}\right]\{Q(x, 0)\} \\
+t a \epsilon_{+-} x\left[y^{1}\right]\{Q(x, y)\}+t a \epsilon_{0-}\left[y^{1}\right]\{Q(x, y)+(b-1) Q(0, y)\} \\
+t a \epsilon_{--} \bar{x}\left[y^{1}\right]\{Q(x, y)-Q(0, y)\}+t a(b-1) \epsilon_{--}\left[x^{1} y^{1}\right]\{Q(x, y)\} .
\end{gathered}
$$

Similarly for walks ending on the $y$-axis:

$$
\begin{gathered}
Q(0, y)=1+t b \epsilon_{0+} y Q(0, y)+t b \epsilon_{0-} \bar{y}(Q(0, y)-Q(0,0))+t(a-1) b \epsilon_{0-}\left[y^{1}\right]\{Q(0, y)\} \\
+t b \epsilon_{-+} y\left[x^{1}\right]\{Q(x, y)\}+t b \epsilon_{-0}\left[x^{1}\right]\{Q(x, y)+(a-1) Q(x, 0)\} \\
+t b \epsilon_{--} \bar{y}\left[x^{1}\right]\{Q(x, y)-Q(x, 0)\}+t(a-1) b \epsilon_{--}\left[x^{1} y^{1}\right]\{Q(x, y)\} .
\end{gathered}
$$

Finally, for walks ending at the corner:

$$
Q(0,0)=1+\operatorname{tab\epsilon }_{-0}\left[x^{1}\right]\{Q(x, 0)\}+\operatorname{tab}_{0-}\left[y^{1}\right]\{Q(0, y)\}+t a b \epsilon_{--}\left[x^{1} y^{1}\right]\{Q(x, y)\} .
$$

Using all of these equations and eliminating all the unknowns except $Q(x, y), Q(x, 0)$, $Q(0, y)$ and $Q(0,0)$ we finally arrive at a functional equation satified by $Q(x, y)$.

Theorem 6. The generating function $Q(x, y)$, with weight a associated with vertices on the $x$-axis and weight $b$ associated with vertices on the $y$-axis, satisfies the functional equation

$$
\begin{array}{r}
x y K(x, y) Q(x, y)=\frac{x y}{a b}+x\left(y-\frac{y}{a}-t A_{-1}(x)\right) Q(x, 0)+y\left(x-\frac{x}{b}-t B_{-1}(y)\right) Q(0, y) \\
-\left(\frac{x y}{a b}(1-a)(1-b)-t \epsilon\right) Q(0,0) .
\end{array}
$$


Proof. We can proceed as described above. Alternatively, we can derive the equation via a double-counting argument, which we give here.

Let

$$
Q^{\dagger}(x, y)=Q(x, y)+\frac{1}{a} Q(x, 0)+\frac{1}{b} Q(0, y)+\left(\frac{1}{a b}-\frac{1}{a}-\frac{1}{b}\right) Q(0,0) .
$$

Observe that $Q^{\dagger}(x, y)$ counts every walk ending on a boundary twice: once in $Q(x, y)$ (with the correct weight), and once in the other terms (underweighted by exactly one factor of $a, b$ or $a b$, depending on whether the walk ends on the $x$-axis, the $y$-axis, or in the corner). Every other walk is counted once with the correct weight (in $Q(x, y)$ ).

Let us now count this same set in another way. Firstly, walks ending on the boundaries (with the correct weights) have generating function

$$
Q(x, 0)+Q(y, 0)-Q(0,0) .
$$

Next, a non-empty walk can be constructed by appending a step to an existing walk, while being careful to subtract off walks that overstep the boundaries:

$$
t\left(S(x, y) Q(x, y)-\bar{y} A_{-1}(x) Q(x, 0)-\bar{x} B_{-1}(y) Q(0, y)+\overline{x y} \epsilon Q(0,0)\right) .
$$

Note that a walk counted by (28) which ends on a boundary will be missing exactly one factor of $a, b$ or $a b$, depending on whether it finishes on the horizontal boundary, vertical boundary or at the corner (respectively). Walks which do not end on a boundary are correctly weighted.

Finally, the empty walk has not yet been counted with the incorrect weight - this is simply $\frac{1}{a b}$. Adding this term to (27) and (28), and equating with $Q^{\dagger}(x, y)$, gives

$$
\begin{aligned}
Q^{\dagger}(x, y)= & Q(x, y)+\frac{1}{a} Q(x, 0)+\frac{1}{b} Q(0, y)+\left(\frac{1}{a b}-\frac{1}{a}-\frac{1}{b}\right) Q(0,0) \\
= & \frac{1}{a b}+Q(x, 0)+Q(y, 0)-Q(0,0) \\
& \quad+t\left(S(x, y) Q(x, y)-\bar{y} A_{-1}(x) Q(x, 0)-\bar{x} B_{-1}(y) Q(0, y)+\overline{x y} \epsilon Q(0,0)\right) .
\end{aligned}
$$

Multiplying both sides by $x y$ and rearranging gives (25).

Notice that equation (25) neatly separates into a bulk term, involving $Q(x, y)$, on the left-hand side, and initial and boundary terms, involving $Q(x, 0), Q(0, y)$ and $Q(0,0)$, on the right-hand side. To solve these equations for different step sets we use the obstinate kernel method [7]. For almost all models we use a half-orbit sum over selected symmetries of the kernel, which allows us to remove selected boundary terms. Then setting the kernel to zero by choosing an appropriate root then eliminates the bulk terms. We then extract the power series $Q(0,0)$ by calculating a constant term. Model 19, Kreweras walks, requires a modified approach — see [8] and Section 8.

The following lemma regarding the roots of the kernel will be useful. For a proof, see Lemma 7 of [9].

Lemma 1. If $A_{-1}(x)$ and $A_{1}(x)$ are both non-zero, then the kernel $K(x, y)=1-t S(x, y)$ has two roots in $y$, exactly one of which has a power series expansion in $t$. 


\section{$3 \quad$ Model 1}

Models 1-4 all have vertical and horizontal symmetry. It turns out that Models 2 and 3 have an additional property which simplifies their solutions, and so we present them in the next section. Model 4 has characteristics of both of these types, and will be covered at the end of the next section.

Here we have $S(x, y)=x+y+\bar{x}+\bar{y}$, so that $A_{-1}(x)=B_{-1}(y)=1$ and $\epsilon=0$. Equation (25) becomes

$$
\begin{aligned}
x y K(x, y) Q(x, y)=\frac{x y}{a b}+x( & \left.-\frac{y}{a}-t\right) Q(x, 0) \\
& +y\left(x-\frac{x}{b}-t\right) Q(0, y)-\frac{x y}{a b}(1-a)(1-b) Q(0,0),
\end{aligned}
$$

where the kernel is

$$
K(x, y)=1-t(x+y+\bar{x}+\bar{y})
$$

As noted in Table 1 , the kernel is symmetric under the involutions $(x, y) \mapsto(\bar{x}, y)$ and $(x, y) \mapsto(x, \bar{y})$. These generate a group of 4 symmetries $\{(x, y),(\bar{x}, y),(x, \bar{y}),(\bar{x}, \bar{y})\}$. The latter three symmetries can be used to construct new equations. We will, shortly, set the kernel to zero by substituting a power series for $y$. Because of this, we will only make use of $\{(x, y),(\bar{x}, y)\}$, since the power series that then result from substituting a power series for $y$ are convergent. If we use $\{(x, \bar{y}),(\bar{x}, \bar{y})\}$, then we cannot make the same substitution for $y$ since the results do not converge inside the ring of formal power series.

Substituting $(x, y) \mapsto(\bar{x}, y)$ into our functional equation gives

$$
\begin{aligned}
\bar{x} y K(x, y) Q(\bar{x}, y)=\frac{\bar{x} y}{a b}+\bar{x}( & \left.y-\frac{y}{a}-t\right) Q(\bar{x}, 0) \\
& +y\left(\bar{x}-\frac{\bar{x}}{b}-t\right) Q(0, y)-\frac{\bar{x} y}{a b}(1-a)(1-b) Q(0,0) .
\end{aligned}
$$

We then eliminate $Q(0, y)$ by taking an appropriate linear combination of equations (30) and (32),

$$
\begin{aligned}
\frac{a y K(x, y)}{a y-t a-y} & \left(Q(x, y)+\frac{\bar{x}(b x-t b-x)}{t b x-b+1} Q(\bar{x}, y)\right)=\frac{t \bar{x} y\left(x^{2}-1\right)}{(a y-t a-y)(t b x-b+1)} \\
+ & Q(x, 0)+\frac{\bar{x}(b x-t b-x)}{t b x-b+1} Q(\bar{x}, 0)-\frac{t \bar{x} y(a-1)(b-1)\left(x^{2}-1\right)}{(a y-t a-y)(t b x-b+1)} Q(0,0) .
\end{aligned}
$$

Let $Y \equiv Y(t ; x)$ be the root of $K(x, y)$ in the variable $y$ which is a power series in $t$. (Recall Lemma 1.)

$$
Y(t ; x)=\frac{x-t-t x^{2}-\sqrt{\left(t-x-2 t x+t x^{2}\right)\left(t-x+2 t x+t x^{2}\right)}}{2 t x}=t+O\left(t^{2}\right) .
$$


Since all unknowns in (33) are power series in $t$ with coefficients that are polynomials in $y$, the substitution $y \mapsto Y$ is valid (ie. all functions converge as power series), and cancels the LHS of (33).

Thus

$$
\begin{aligned}
& 0=\frac{t \bar{x} Y\left(x^{2}-1\right)}{(a Y-t a-Y)(t b x-b+1)}+Q(x, 0) \\
& \quad+\frac{\bar{x}(b x-t b-x)}{t b x-b+1} Q(\bar{x}, 0)-\frac{t \bar{x} Y(a-1)(b-1)\left(x^{2}-1\right)}{(a Y-t a-Y)(t b x-b+1)} Q(0,0) .
\end{aligned}
$$

Notice that the coefficient of $Q(\bar{x}, 0)$ is a rational function of $b, x$ and $t$. This fact is intriguing but at this point we have no combinatorial explanation. Regardless, it does now allow us to compute a required constant term in closed form as we now demonstrate.

\subsection{General $a, b$ directly}

Notice that the first and last terms in equation (35) are nearly identical. Grouping them together gives

$$
\begin{aligned}
0=\frac{t \bar{x} Y\left(x^{2}-1\right)}{(a Y-t a-Y)(t b x-b+1)}(1-(a-1)(b-1) Q(0,0)) & \\
& +Q(x, 0)+\frac{\bar{x}(b x-t b-x)}{t b x-b+1} Q(\bar{x}, 0) .
\end{aligned}
$$

Write

$$
C=\left[x^{0}\right]\left\{\frac{t \bar{x} Y\left(x^{2}-1\right)}{(a Y-t a-Y)(t b x-b+1)}\right\},
$$

then the coefficient of $x^{0}$ of the first term is

$$
C \cdot(1-(a-1)(b-1) Q(0,0)) .
$$

The constant term of the second term is just $Q(0,0)$.

As remarked above, the rationality of the coefficient of $Q(\bar{x}, 0)$ allows us to compute the required constant term. The third term can be viewed as the product of two power series in $t$ :

$$
\begin{aligned}
\frac{b x-b t-x}{(b x t-b+1) x} & =-\frac{1}{x^{2}}+\frac{1-x^{2}}{x^{2}} \sum_{n \geqslant 0}\left(\frac{b x}{b-1}\right)^{n} t^{n} \\
& =-\frac{1}{x^{2}}-\sum_{n \geqslant 0}\left(\frac{t b}{b-1}\right)^{n}\left(x^{n}-x^{n-2}\right)
\end{aligned}
$$

and

$$
Q(\bar{x}, 0)=\sum_{m, k \geqslant 0} q_{m, k, 0} t^{m} x^{-k}
$$


When we take the product and extract the constant term, the only terms that are left are

$$
\begin{aligned}
-\sum_{m, k \geqslant 0} q_{m, k, 0} t^{n}\left(\frac{t b}{b-1}\right)^{k}+\sum_{m, k \geqslant 0} q_{m, k, 0} t^{n}\left(\frac{t b}{b-1}\right)^{k+2} & \\
& =-\left(1-\left(\frac{b t}{b-1}\right)^{2}\right) Q\left(\frac{t b}{b-1}, 0\right) .
\end{aligned}
$$

Reassembling these components we have

$$
0=C \cdot(1-(a-1)(b-1) Q(0,0))+Q(0,0)-\left(1-\left(\frac{b t}{b-1}\right)^{2}\right) Q\left(\frac{t b}{b-1}, 0\right) .
$$

At this point, it appears that we have just replaced $Q(\bar{x}, 0)$ with a new unknown $Q\left(\frac{t b}{b-1}, 0\right)$. In fact, this new unknown can be expressed in terms of $Q(0,0)$ by careful manipulation of the original functional equation. Substituting $x \mapsto \frac{t b}{b-1}$ in (30) we get

$$
\begin{aligned}
\frac{t b y}{b-1} K\left(\frac{t b}{b-1}\right. & , y) Q\left(\frac{t b}{b-1}, y\right) \\
& =\frac{t y}{a(b-1)}+\frac{t b}{b-1}\left(y-\frac{y}{a}-t\right) Q\left(\frac{t b}{b-1}, 0\right)+\frac{t y}{a}(1-a) Q(0,0) .
\end{aligned}
$$

Note, in particular, that the $Q(0, y)$ term has been cancelled. The new kernel $K\left(\frac{t b}{b-1}, y\right)$ has a power series root in $y$ :

$$
Y^{\prime}(t ; b)=Y\left(t ; \frac{t b}{b-1}\right)
$$

Substituting $y \mapsto Y^{\prime}$, we have

$$
0=\frac{Y^{\prime}}{a(b-1)}+\frac{b}{b-1}\left(Y^{\prime}-\frac{Y^{\prime}}{a}-t\right) Q\left(\frac{t b}{b-1}, 0\right)+\frac{Y^{\prime}}{a}(1-a) Q(0,0) .
$$

Now isolate $Q\left(\frac{t b}{b-1}, 0\right)$ :

$$
\begin{aligned}
Q\left(\frac{t b}{b-1}, 0\right) & =-\frac{Y^{\prime}}{b\left(a Y^{\prime}-Y^{\prime}-t a\right)}(1-(a-1)(b-1) Q(0,0)) \\
& \equiv-P \cdot(1-(a-1)(b-1) Q(0,0))
\end{aligned}
$$

Substituting this into (42) and solving for $Q(0,0)$ gives

$$
\begin{aligned}
G(t ; a, b)= & Q(0,0) \\
= & \frac{1}{(a-1)(b-1)} \\
& \quad+\frac{1}{(a-1)(b-1)+(a-1)^{2}(b-1)^{2} C-(a-1)^{2}(t b-b+1)(t b+b-1) P} .
\end{aligned}
$$


The first few terms are

$$
\begin{aligned}
Q(0,0)=1+ & \left(a^{2} b+a b^{2}\right) t^{2} \\
& +\left(a^{3} b+a^{4} b+2 a^{2} b^{2}+a^{4} b^{2}+a b^{3}+2 a^{3} b^{3}+a b^{4}+a^{2} b^{4}\right) t^{4}+O\left(t^{6}\right) .
\end{aligned}
$$

Since $P$ is algebraic and $C$ is D-finite, $Q$ is at worst D-algebraic.

We note here that (43) and (46) may have some combinatorial interpretation - for example, $Q\left(\frac{t b}{b-1}, 0\right)$ can be viewed as the generating function of walks ending on the $x$ axis, where each walk ending at $(k, 0)$ has $k$ west steps appended, each picking up weight $\frac{b}{b-1}$ (and not weight $a$ ). How to write this in terms of $Q(0,0)$ (and thus interpret (46)) is not clear to us.

\subsection{General $a, b$ via $a=1$ and $b=1$}

We now present a similar solution to the model which uncovers more structure of the problem, at the expense of being less direct. In particular we find an expression for $G(t ; a, b)$ in terms of $G(t ; a, 1)$ and $G(t ; 1, b)$. These two subproblems are significantly easier to solve. Let $P(x, y)=\left.Q(x, y)\right|_{b=1}$ and $R(x, y)=\left.Q(x, y)\right|_{a=1}$. We first solve for $P$ and then $R$ follows immediately by symmetry.

When $b=1$ the functional equation and its solution simplify dramatically. Equation (35) becomes

$$
0=\frac{\bar{x}^{2} Y\left(x^{2}-1\right)}{a Y-t a-Y}+P(x, 0)-\bar{x}^{2} P(\bar{x}, 0)
$$

and hence

$$
P(0,0)=-\left[x^{0}\right]\left\{\frac{\bar{x}^{2} Y\left(x^{2}-1\right)}{a Y-t a-Y}\right\} .
$$

This is a D-finite function. By symmetry we then have the solution for $R(0,0)$.

We now show that the solution for general $a, b$ can be written in terms of $P$ and $R$. Rearrange (35) once more:

$$
\begin{aligned}
0=\frac{t \bar{x}^{2} Y\left(x^{2}-1\right)}{(a Y-t a-Y)} & +\bar{x}(t b x-b+1) Q(x, 0) \\
& +\bar{x}^{2}(b x-t b-x) Q(\bar{x}, 0)-\frac{t \bar{x}^{2} Y(a-1)(b-1)\left(x^{2}-1\right)}{a Y-t a-Y} Q(0,0) .
\end{aligned}
$$

Notice that the $Q$-independent term is precisely $t$ times the $P$-independent term in (51). Hence its constant term is exactly $t P(0,0)$. Consequently the constant term of the whole equation is

$$
0=-t P(0,0)+t b Q(0,0)-(b-1)\left[x^{1}\right]\{Q(x, 0)\}+t(a-1)(b-1) P(0,0) Q(0,0) .
$$

By symmetry,

$$
0=-t R(0,0)+t a Q(0,0)-(a-1)\left[y^{1}\right]\{Q(0, y)\}+t(a-1)(b-1) R(0,0) Q(0,0) .
$$


At this point we have introduced two new unknowns $\left[x^{1}\right]\{Q(x, 0)\}$ and $\left[y^{1}\right]\{Q(0, y)\}$. These can be eliminated using the following relation

$$
Q(0,0)=1+\operatorname{tab}\left[x^{1}\right]\{Q(x, 0)\}+\operatorname{tab}\left[y^{1}\right]\{Q(0, y)\},
$$

which is equation (24) for Model 1 . This equation simply says that any walk ending in the corner either has length 0 or arrives by taking a step south or west. This system of three equations can be solved to find $Q(0,0)$ in terms of $P \equiv P(0,0)$ and $R \equiv R(0,0)$ :

$$
\begin{aligned}
& Q(0,0)= \\
& \quad \frac{(1-a)(1-b)-t^{2} a b((a-1) P+(b-1) R)}{(1-a)(1-b)-t^{2} a b(2 a b-a-b)-t^{2} a b(a-1)(b-1)((a-1) P+(b-1) R)} .
\end{aligned}
$$

When $a=1$ or $b=1$, this simply reduces to $R(0,0)$ and $P(0,0)$ respectively. Also the above expression can be written in partial fraction form so that the numerator is free of $P$ and $R$. The solution of Model 22 [28] has a very similar form.

There may be a purely combinatorial interpretation of $(57)$ - for example, note that by translating walks one unit east or north, we see that $P$ and $R$ are (essentially) the generating functions of walks which never touch the $y$ - and $x$-axes respectively. Equation (57) suggests there is some way of combining these to get the full generating function, but we have not been able to find it.

\section{Models 2, 3 and 4}

\subsection{Model 2 via the Hadamard product of Dyck paths}

Model 2 has horizontal and vertical symmetry but is different from Models 1 and 4 in that it has a D-finite generating function for all $a, b$ (Model 3 is also D-finite).

Before tackling the functional equation, there is a very elegant solution that comes from the observation that $G(t ; 1,1)$ can be written as the Hadamard product of the generating function of Dyck paths with itself. To see this, note that any walk starting and ending at the origin can be factored into a pair of Dyck paths. More precisely, the $x$-coordinate (and $y$-coordinate) for any such walk of length $2 n$ is a sequence of non-negative integers starting and ending at 0 , with steps \pm 1 . The number of such sequences is given by the $n^{\text {th }}$ Catalan number $c_{n}=\frac{1}{n+1}\left(\begin{array}{c}2 n \\ n\end{array}\right)$. Since these two sequences are completely independent we have that the number of such paths (ignoring, for the moment visits to either wall) is just $c_{n}^{2}$.

This reasoning continues to hold when visits to either wall are counted, and one obtains

$$
\left[t^{n}\right]\{Q(0,0)\}=\left[t^{n}\right]\{G(t ; a, b)\}=Z_{n}(a) Z_{n}(b),
$$

where $Z_{n}(a)$ is the coefficient of $t^{n}$ in

$$
f(t ; a)=\frac{2}{2-a+a \sqrt{1-4 t^{2}}}=\sum_{n \geqslant 0} t^{2 n} \sum_{k=1}^{n} \frac{k}{n}\left(\begin{array}{c}
2 n-k-1 \\
n-k
\end{array}\right) a^{k},
$$


being the generating function of Dyck paths counted by their length and number of visits to the axis. This generating function is classical and can be quickly established via a factorisation argument which gives the functional equation

$$
f(t ; a)=1+t^{2} f(t ; 1) f(t ; a) .
$$

Consequently the generating function $Q(0,0)$ can be written as the Hadamard product

$$
Q(0,0)=G(t ; a, b)=f(t ; a) \odot_{t} f(t ; b)=\sum_{n \geqslant 0} t^{n}\left(\left[t^{n}\right]\{f(t, a)\}\right)\left(\left[t^{n}\right]\{f(t, b)\}\right) .
$$

Since D-finite functions are closed under Hadamard products [21], the above is D-finite. Note that it cannot be algebraic, because when $a, b=1$, the coefficients of $Q(0,0)$ grow as $16^{n} / n^{3}$, a form that is incompatible with algebraic generating functions - see, for example, Section VII.7 of [12].

Model 3 is similar to Model 2 in that its solution can also be written as a Hadamard product of D-finite functions - see Section 4.3 below.

\subsection{Model 2 via the kernel method}

We can also show that the solution is D-finite via the functional equation using the kernel method. The functional equation is

$$
\begin{aligned}
x y K(x, y) Q(x, y) & =\frac{x y}{a b}+x\left(y-\frac{y}{a}-t(x+\bar{x})\right) Q(x, 0) \\
+ & y\left(x-\frac{x}{b}-t(y+\bar{y})\right) Q(0, y)-\left(\frac{x y}{a b}(1-a)(1-b)-t\right) Q(0,0),
\end{aligned}
$$

where

$$
K(x, y)=1-t(x y+x \bar{y}+\bar{x} y+\overline{x y}) .
$$

As noted in Table 1, the kernel is invariant under the involutions $(x, y) \mapsto(\bar{x}, y),(x, \bar{y})$. Proceeding as for Model 1, we map $(x, y) \mapsto(\bar{x}, y)$,

$$
\begin{aligned}
& \bar{x} y K(x, y) Q(\bar{x}, y)=\frac{\bar{x} y}{a b}+\bar{x}\left(y-\frac{y}{a}-t(x+\bar{x})\right) Q(\bar{x}, 0) \\
&+y\left(\bar{x}-\frac{\bar{x}}{b}-t(y+\bar{y})\right) Q(0, y)-\left(\frac{\bar{x} y}{a b}(1-a)(1-b)-t\right) Q(0,0) .
\end{aligned}
$$

Eliminating $Q(0, y)$ between the two functional equations we have,

$$
\begin{aligned}
\frac{a x y K(x, y)}{t a+t a x^{2}+x y-a x y}\left(-Q(x, y)+\frac{\bar{x}\left(t b+x y-b x y+t b y^{2}\right)}{t b x+y-b y+t b x y^{2}} Q(\bar{x}, y)\right) \\
=-\frac{t y\left(x^{2}-1\right)\left(1+y^{2}\right)}{\left(t a+t a x^{2}+x y-a x y\right)\left(t b x+y-b y+t b x y^{2}\right)}+Q(x, 0) \\
\quad-\frac{\bar{x}\left(t b+x y-b x y+t b y^{2}\right)}{t b x+y-b y+t b x y^{2}} Q(\bar{x}, 0) \\
+\frac{t y(b-1)\left(x^{2}-1\right)\left(a y^{2}-y^{2}-1\right)}{\left(t a+t a x^{2}+x y-a x y\right)\left(t b x+y-b y+t b x y^{2}\right)} Q(0,0) .
\end{aligned}
$$


As for Model 1, we set the kernel to zero by substituting $y=Y(t ; x)$ being a power series solution to $K(x, y)=0$. After simplifying the coefficients we arrive at

$$
\begin{aligned}
& 0=-\frac{x Y\left(x^{2}-1\right)}{\left(1+x^{2}-b\right)\left(t a+t a x^{2}+x Y-a x Y\right)} \\
& +Q(x, 0)-\frac{1+x^{2}-b x^{2}}{1+x^{2}-b} Q(\bar{x}, 0)-\frac{(b-1)\left(x^{2}-1\right)}{1+x^{2}-b} Q(0,0) .
\end{aligned}
$$

The first term in (66) can be viewed as a power series in $t$ with coefficients that are Laurent polynomials in $x$; it thus has a well-defined constant term with respect to $x$ (see (69) below).

Now the coefficient of $Q(\bar{x}, 0)$ does not depend on $t$, so expanding everything as a power series in $t$ and taking the constant term with respect to $x$ is not an option here. We are left to consider things in terms of $x$ or, alternatively, $\bar{x}$.

Expanding in $\bar{x}$, things are straightforward. Since

$$
\begin{gathered}
-\frac{1+x^{2}-b x^{2}}{1+x^{2}-b}=(b-1)+b(b-2) \bar{x}^{2}+O\left(\bar{x}^{4}\right), \text { and } \\
-\frac{(b-1)\left(x^{2}-1\right)}{1+x^{2}-b}=-(b-1)-(b-1)(b-2) \bar{x}^{2}+O\left(\bar{x}^{4}\right),
\end{gathered}
$$

the third and fourth terms cancel, leaving

$$
Q(0,0)=\left[x^{0}\right]\left\{\frac{x Y\left(x^{2}-1\right)}{\left(1+x^{2}-b\right)\left(t a+t^{2} x^{2}+x Y-a x Y\right)}\right\} .
$$

This shows that $Q(0,0)$ is D-finite. We note that if one expands in $x$ rather than $\bar{x}$ then one can arrive, with more work, at a similar though less appealing D-finite expression.

At first it may seem that the method used in Section 3.2 could be applied to Model 2 , but unfortunately we have not been able to make this work. It is straightforward to solve $P(0,0)$ and $R(0,0)$ as constant terms of algebraic functions (similarly to (52)), and to derive the equivalents of (54) and(55):

$$
\begin{aligned}
& 0=P(0,0)-Q(0,0)+(b-1)\left[x^{2}\right]\{Q(x, 0)\} \\
& 0=R(0,0)-Q(0,0)+(a-1)\left[y^{2}\right]\{Q(0, y)\} .
\end{aligned}
$$

The problem is that the equivalent of (56) is now

$$
Q(0,0)=1+t^{2} a b\left(Q(0,0)+\left[x^{2}\right]\{Q(x, 0)\}+\left[y^{2}\right]\{Q(0, y)\}+\left[x^{2} y^{2}\right]\{Q(x, y)\}\right)
$$

and there is no way to eliminate the $\left[x^{2} y^{2}\right]\{Q(x, y)\}$ term.

Of course this does not mean that there is no way to write $Q(0,0)$ in terms of $P(0,0)$ and $R(0,0)$ - but if there is, we do not know how to do it. 


\section{3 $\quad$ Model 3}

Both of the methods presented above for solving Model 2 can also be applied to Model 3. For the first method - taking a Hadamard product - instead of two Dyck paths we now have a Dyck path with a Motzkin path. This is because the $y$-coordinate is still a sequence of non-negative integers starting and ending at 0 , with steps \pm 1 , while for the $x$-coordinate the steps are $0, \pm 1$. As a result we have (analogously to (58))

$$
\left[t^{n}\right]\{Q(0,0)\}=\left[t^{n}\right]\{G(t ; a, b)\}=Z_{n}(a) Y_{n}(b),
$$

where $Z_{n}(a)$ is as per (59), and $Y_{n}(b)$ is the coefficient of $t^{n}$ in

$$
g(t ; b)=\frac{2}{2-b-b t+b \sqrt{(1+t)(1-3 t)}},
$$

the generating function for Motzkin paths which accrue weight $b$ with each visit to the boundary. So the generating function $Q(0,0)$ can be written as the Hadamard product

$$
Q(0,0)=G(t ; a, b)=f(t, a) \odot_{t} g(t, b) \sum_{n \geqslant 0} t^{n}\left(\left[t^{n}\right]\{f(t, a)\}\right)\left(\left[t^{n}\right]\{g(t, b)\}\right)
$$

and consequently $Q(0,0)$ is D-finite. Similarly to Model $2, Q(0,0)$ cannot be algebraic.

The second method - using the functional equation - is very similar to what was done for Model 2. The analogue of (66) is

$$
\begin{aligned}
0=\frac{t\left(1-x^{2}\right) Y\left(1+Y^{2}\right)}{\left(t a+t a x+t a x^{2}+x Y-a x Y\right)\left(t x b+Y-b Y+t b x Y^{2}\right)}+Q(x, 0) \\
\quad-\frac{1+x-b x+x^{2}-b x^{2}}{1-b+x-b x+x^{2}} Q(\bar{x}, 0)+\frac{(b-1)\left(1-x^{2}\right)}{1-b+x-b x+x^{2}} Q(0,0)
\end{aligned}
$$

where $Y$ is a root of the kernel $K(x, y)=1-t(x y+y+\bar{x} y+\overline{x y}+\bar{y}+x \bar{y})$. Expanding the coefficients of $Q(\bar{x}, 0)$ and $Q(0,0)$ in $\bar{x}$ reveals that their contributions to the constant term cancel (analogously to (67) and (68)), and as a result

$$
Q(0,0)=\left[x^{0}\right]\left\{-\frac{t\left(1-x^{2}\right) Y\left(1+Y^{2}\right)}{\left(t a+t a x+t a x^{2}+x Y-a x Y\right)\left(t x b+Y-b Y+t b x Y^{2}\right)}\right\} .
$$

As with Model 2, it is also possible to obtain the solution by expanding those coefficients in $x$ instead of $\bar{x}$, but considerably more work is required.

\subsection{Model 4}

This model can be solved by using (at least) two of the above methods. The analogue of (35) has the form

$$
0=C+Q(x, 0)-\frac{1+t b+x-b x+x^{2}-b x^{2}}{1-b+x-b x+x^{2}+t b x^{2}} Q(\bar{x}, 0)+C_{0,0} Q(0,0)
$$


where $C$ and $C_{0,0}$ are algebraic (being rational functions of $t, x, a, b$ and the kernel root $Y)$. Then things can be expanded in $x$ or $\bar{x}$. For the former the methodology resembles that of Section 3.1, except things are more complicated because the coefficient of $Q(\bar{x}, 0)$ must be split apart using partial fractions. For the latter, things are somewhat simpler the contribution of the $Q(\bar{x}, 0)$ term ends up being $\frac{b-1}{1+t b} Q(0,0)$.

In either case one is left with an equation involving $Q(0,0)$ and the constant terms of algebraic things. The solution is thus the ratio of D-finite functions, and is hence D-algebraic.

If $a=1$ or $b=1$ then things simplify dramatically, in a manner very similar to Model 1. In those cases the solution is D-finite.

\section{D-finite solution to horizontally symmetric models}

Models 1 through 16 all possess symmetry across a vertical line, and we have stated that all of them are D-finite when $b=1$. We prove that this is the case using a variation of Bousquet-Mélou's argument from [7]. Let us start by giving the functional equation satisfied by these models when $b=1$ :

$$
x y K(x, y) Q(x, y)=\frac{x y}{a}+x\left(y-\frac{y}{a}-t A_{-1}(x)\right) Q(x, 0)-t y B_{-1}(y) Q(0, y)+t \epsilon Q(0,0) .
$$

Since the step set is symmetric across a vertical line, the kernel is symmetric under the involution $(x, y) \mapsto(\bar{x}, y)$. Using that substitution we obtain

$$
\bar{x} y K(x, y) Q(\bar{x}, y)=\frac{y}{a x}+\bar{x}\left(y-\frac{y}{a}-t A_{-1}(x)\right) Q(\bar{x}, 0)-t y B_{-1}(y) Q(0, y)+t \epsilon Q(0,0),
$$

where we have used the fact that $A_{-1}(\bar{x})=A_{-1}(x)$ (thanks to the symmetry of the step set). Subtracting one equation from the other eliminates the $Q(0, y)$ term:

$$
\begin{aligned}
K(x, y)(x y Q(x, y)-\bar{x} y Q & (\bar{x}, y)) \\
& =\frac{y(x-\bar{x})}{a}+\left(y-\frac{y}{a}-t A_{-1}(x)\right)(x Q(x, 0)-\bar{x} Q(\bar{x}, 0)) .
\end{aligned}
$$

We can now set the kernel to zero by setting $y=Y(t ; x) \equiv Y(x)$, being the power series solution of $K(x, y)=0$. After a little rearrangement we have

$$
Q(x, 0)-\bar{x}^{2} Q(\bar{x}, 0)=\frac{Y(x)\left(1-\bar{x}^{2}\right)}{Y(x)(1-1 / a)-t A_{-1}(x)} .
$$

Now we are in familiar territory and we proceed by extracting constant term with respect to $x$ from both sides:

$$
Q(0,0)=G(t ; a, 1)=\left[x^{0}\right]\left\{\frac{Y(x)\left(1-\bar{x}^{2}\right)}{a t A_{-1}(x)+(1-a) Y(x)}\right\} .
$$


This shows that $Q(0,0)$ is D-finite. We note that when $a=b=1$ none of Models 1 through 16 have an algebraic generating function, and so none of these models have an algebraic generating function when $b=1$.

\section{$6 \quad$ Model 17}

The functional equation and kernel of Model 17 are:

$$
\begin{aligned}
& \begin{aligned}
a b x y K(x, y) Q(x, y)= & x y+b x(a y-y-a x t) Q(x, 0) \\
& +a y(b x-x-b t) Q(0, y)-(a-1)(b-1) x y Q(0,0),
\end{aligned} \\
& \text { where } K(x, y)=1-t(y+\bar{x}+x \bar{y}) .
\end{aligned}
$$

As noted in Table 4, the kernel is symmetric under involutions $(x, y) \mapsto(\bar{x} y, y)$ and $(x, y) \mapsto(x, x \bar{y})$. This gives a group of 6 symmetries, which generate 5 new equations. Let us start by using those obtained by setting $(x, y)=(\bar{x} y, y),(\bar{x} y, \bar{x})$ :

$$
\begin{aligned}
a b \bar{x} y^{2} K(\bar{x} y, y) Q(x, y)= & \bar{x} y^{2}+b \bar{x}^{2} y^{2}(a x-x-a t) Q(\bar{x} y, 0) \\
& +a \bar{x} y(b y-y-b x t) Q(0, y)-(a-1)(b-1) \bar{x} y^{2} Q(0,0)
\end{aligned}
$$

and

$$
\begin{aligned}
a b x y K(x, y) Q(\bar{x} y, \bar{x})= & \bar{x}^{2} y+b \bar{x}^{2} y(a-1-a y t) Q(\bar{x} y, 0) \\
& +a \bar{x}^{2}(b y-y-b x t) Q(0, \bar{x})-(a-1)(b-1) \bar{x}^{2} y Q(0,0) .
\end{aligned}
$$

We can set the kernel to zero in all of these by choosing $Y=Y(x)$, being the power series solution to $K(x, y)=0$. This leaves only the right-hand side of the 3 equations. Then taking a linear combination of the equations we eliminate the $Q(0, y)$ and $Q(\bar{x} y, 0)$ terms, and after a little massaging of the result we have

$$
\begin{aligned}
& Q(x, 0)+\frac{a(t b-b x+x)(a t-a x+x) Y}{x^{2}(Y a t-a+1)(-t x a+Y a-Y) b} Q(0, \bar{x}) \\
&=H(x, Y(x) ; a, b)(1-Q(0,0)(a-1)(b-1))
\end{aligned}
$$

for a function $H$ which is rational in its arguments.

With a little algebra one can verify that the coefficient of $Q(0, \bar{x})$ is actually a rational function of $t, x, a, b$ :

$$
\frac{a(t b-b x+x)(a t-a x+x)}{\left(x^{2} t^{2} a^{2}+t a^{2}-a t-a x+x\right) x b} .
$$

Before solving equation (88) in generality, let us first verify that when $a=1$ we obtain a D-finite solution. 


\subsection{An aside to $a=1$}

Note that equation (88) simplifies considerably when $a=1$ to give

$$
Q(x, 0)+\frac{t b x-b x+x}{x^{3}} Q(0, \bar{x})=\frac{Y\left(Y b t x-b t x^{3}-b t+b x-x\right)}{t b x^{3}(Y b-b t x-Y)} .
$$

Extracting the constant term with respect to $x$ of both sides then yields

$$
\left.Q(0,0)\right|_{a=1}=G(t ; 1, b)=\left[x^{0}\right]\left\{\frac{Y\left(Y b t x-b t x^{3}-b t+b x-x\right)}{t b x^{3}(Y b-b t x-Y)}\right\} .
$$

This demonstrates that when $a=1 Q(0,0)$ is D-finite.

\subsection{Back to general $(a, b)$}

We rewrite equation (88) with the coefficient of $Q(0, \bar{x})$ made explicitly rational:

$$
\begin{aligned}
& Q(x, 0)+\frac{a(t b-b x+x)(a t-a x+x)}{\left(x^{2} t^{2} a^{2}+t a^{2}-a t-a x+x\right) x b} Q(0, \bar{x}) \\
& \quad=H(x, Y(x) ; a, b)(1-Q(0,0)(a-1)(b-1)) .
\end{aligned}
$$

We proceed as per the $a=1$ case, by taking the constant term of the above with respect to $x$. The main difficulty now lies in computing

$$
\left[x^{0}\right]\left\{\frac{a(t b-b x+x)(a t-a x+x)}{\left(x^{2} t^{2} a^{2}+t a^{2}-a t-a x+x\right) x b} Q(0, \bar{x})\right\} .
$$

Write the coefficient of $Q(0, \bar{x})$ as

$$
C_{0}+\frac{C_{1}}{x}+\frac{C_{2}}{1-x / \xi_{+}}+\frac{C_{3}}{1-\bar{x} \cdot \xi_{-}}
$$

where $\xi_{ \pm}$are solutions of the denominator factor $x^{2} t^{2} a^{2}+t a^{2}-a t-a x+x$ :

$$
\begin{aligned}
& \xi_{+}=\frac{(a-1)+\sqrt{(1-a)\left(1-a+4 a^{3} t^{3}\right)}}{2 a^{2} t^{2}}=\frac{a-1}{a^{2}} t^{-2}+O(t) ; \\
& \xi_{-}=\frac{(a-1)-\sqrt{(1-a)\left(1-a+4 a^{3} t^{3}\right)}}{2 a^{2} t^{2}}=a t+O\left(t^{4}\right) .
\end{aligned}
$$

This particular combination of $x, \bar{x}, \xi_{ \pm}$was chosen in (94) so that the expression is a power series in $t$. The coefficient $C_{1}=\frac{a t}{a-1}$ while the others are somewhat messy algebraic functions of $a, b$ and $t$. The desired constant term can now be written quite cleanly in terms of the $C_{i}$ :

$$
\left[x^{0}\right]\left\{\left(C_{0}+\frac{C_{1}}{x}+\frac{C_{2}}{1-x / \xi_{+}}-\frac{C_{0}}{1-\bar{x} \cdot \xi_{-}}\right) Q(0, \bar{x})\right\}=C_{2} \cdot Q\left(0,1 / \xi_{+}\right),
$$


where we have used the fact that $C_{0}=-C_{3}$. This transforms equation (92) into

$$
Q(0,0)+C_{2} Q\left(0,1 / \xi_{+}\right)=(1-Q(0,0)(a-1)(b-1)) \cdot\left[x^{0}\right]\{H(x, Y(x) ; a, b)\} .
$$

Thankfully we can now compute $Q(0,1 / \xi+)$ in terms of $Q(0,0)$ after a little work on the original functional equation (84).

Notice that we can eliminate $Q(x, 0)$ from equation (84) by setting $y=\frac{a x t}{a-1}$. This yields

$$
\begin{aligned}
a b x K\left(x, \frac{a x t}{a-1}\right) Q\left(x, \frac{a x t}{a-1}\right) & = \\
& x+a(b x-x-b t) Q\left(0, \frac{a x t}{a-1}\right)-(a-1)(b-1) x Q(0,0) .
\end{aligned}
$$

We now choose $x$ to set $K\left(x, \frac{a x t}{a-1}\right)$ to zero. The solutions are precisely $x=\xi_{ \pm}$. Since $\xi_{-}=O(t)$ we set $x=\xi_{-}$in (98). Notice that $\frac{a \xi_{-} t}{a-1}=1 / \xi_{+}$, and so we have (after a little manipulation)

$$
Q\left(0,1 / \xi_{+}\right)=\frac{\xi_{-}}{a\left(b \xi_{-}-\xi_{-}-b t\right)}((a-1)(b-1) Q(0,0)-1) .
$$

Substitute this back into equation (98):

$$
\begin{aligned}
Q(0,0)+\frac{C_{2} \xi_{-}}{a\left(b \xi_{-}-\xi_{-}-b t\right)} & ((a-1)(b-1) Q(0,0)-1) \\
= & (1-Q(0,0)(a-1)(b-1)) \cdot\left[x^{0}\right]\{H(x, Y(x) ; a, b)\} .
\end{aligned}
$$

Finally, we can isolate $Q(0,0)$ :

$$
Q(0,0)=\frac{P(t ; a, b)}{1+(a-1)(b-1) P(t ; a, b)},
$$

where

$$
P(t ; a, b)=\frac{C_{2} \xi_{-}}{a\left((b-1) \xi_{-}-b t\right)}-\left[x^{0}\right]\{H(x, Y(x) ; a, b)\} .
$$

Since $P(t ; a, b)$ is D-finite, $Q(0,0)$ is, at worst, D-algebraic. Unfortunately this does not prove that $Q(0,0)$ is not D-finite. Additionally, the series $P(t ; a, b)$ is singular when $a=1$ or $b=1$.

\subsection{Aside to $b=1$}

While we cannot substitute $b=1$ directly into the above expression for $Q(0,0)$ we can recycle most of our workings. Set $b=1$ into equation (92) and take the constant term:

$$
Q(0,0)+\left[x^{0}\right]\left\{\frac{a t(a t-a x+x)}{\left(x^{2} t^{2} a^{2}+t a^{2}-a t-a x+x\right) x} Q(0, \bar{x})\right\}=\left[x^{0}\right] H(x, Y(x) ; a, 1) .
$$


We can then compute the constant term on the left-hand side by very similar methods and demonstrate that

$$
\left[x^{0}\right]\left\{\frac{a t(a t-a x+x)}{\left(x^{2} t^{2} a^{2}+t a^{2}-a t-a x+x\right) x} Q(0, \bar{x})\right\}=\left.C_{2}\right|_{b=1} \cdot Q\left(0,1 / \xi_{-}\right) .
$$

Moreover, by (100), when $b=1$ we have

$$
Q\left(0,1 / \xi_{+}\right)=\frac{\xi_{-}}{a t}
$$

Hence

$$
\left.Q(0,0)\right|_{b=1}=\left[x^{0}\right] H(x, Y(x) ; a, 1)-\left.\frac{\xi_{-}}{a t} \cdot C_{2}\right|_{b=1}
$$

and so is D-finite.

\section{$7 \quad$ Model 18}

We have been unable to solve this model for general $a, b$, however we have been able to solve it along the line $a=b$. As remarked above, this model is very similar to that studied in [29]. When $a=b$, the generating function is symmetric so that $Q(x, y)=Q(y, x)$, and we start by writing the functional equation and associated kernel:

$$
\begin{aligned}
& a b x y K(x, y) Q(x, y)=x y+a x(y(1-a)+a t(x+1)) L(x) \\
& +a y(x(1-a)+a t(y+1)) L(y)-(a-1)^{2} x y L(0) \\
& \text { and } K(x, y)=1-t(x+\bar{x}+y+\bar{y}+x \bar{y}+y \bar{x}) \text {, }
\end{aligned}
$$

where we have written $Q(x, 0)=L(x), Q(0, y)=L(y)$ and $Q(0,0)=L(0)$.

As noted in Table 4 , the kernel admits 6 symmetries which generate 5 new equations; we use those obtained by setting $(x, y)=(x, y),(\bar{x} y, y)$ and $(\bar{x} y, \bar{x})$. An appropriate linear combination of these equations allows us to eliminate the unknown functions $L(y)$ and $L(\bar{x} y)$. We can then eliminate the bulk terms by setting the kernel to zero by substituting $y=Y(t ; x) \equiv Y(x)$ being the power series solution of $K(x, y)=0$ :

$$
\begin{aligned}
Y(t ; x) & \equiv Y(x) \\
& =\frac{x-t-t x^{2}-\sqrt{\left(x^{4}-4 x^{3}-6 x^{2}-4 x+1\right) t^{2}-2 x\left(x^{2}+1\right) t+x^{2}}}{2 t(1+x)} \\
& =O(t) .
\end{aligned}
$$

This then gives

$$
\begin{aligned}
L(x)+\frac{(Y a t+t a-x a+x)(x a t+Y a t-Y x a+Y x)}{(Y a t x+x a t-a Y+Y)(x a t+Y a t-a+1) x^{2}} L(\bar{x}) & \\
& =H(x, Y(x) ; a)\left(1-L(0)(a-1)^{2}\right),
\end{aligned}
$$


where $H$ is a somewhat complicated function that is rational in its arguments. To arrive at the solution we take the constant term of the above equation.

The constant term of $L(x)$ is simply $L(0)$. We can write the constant term of the right-hand side also in terms of $L(0)$ :

$$
\left(1-L(0)(a-1)^{2}\right) \cdot\left[x^{0}\right]\{H(x, Y(x) ; a)\} .
$$

The remaining term is more challenging.

As has been the case in many of the models we have discussed, one can show, with a little work, that the coefficient of $L(\bar{x})$ is actually a rational function of $t, x, a$ :

$$
\frac{(a-1)(t a+a-1) x^{2}-(a-1)(2 t a+1) x+t a(2 t a+1)}{x\left(t a(2 t a+1) x^{2}-(a-1)(2 t a+1) x+(a-1)(t a+a-1)\right)} .
$$

We are able to write it in a partial fraction form:

$$
C_{0}+\frac{C_{1}}{x}+\frac{C_{2}}{1-x / \xi_{+}}+\frac{C_{3}}{1-\bar{x} \xi_{-}},
$$

where the $\xi_{ \pm}$are solutions of the denominator with respect to $x$ :

$$
\begin{aligned}
\xi_{+} & =\frac{2 t a^{2}-2 t a+a-1+\sqrt{-(a-1)(2 t a+1)\left(4 t^{2} a^{2}+2 t a^{2}-2 t a-a+1\right)}}{2 t a(2 t a+1)} \\
& =O\left(t^{-1}\right) ; \\
\xi_{-} & =\frac{2 t a^{2}-2 t a+a-1-\sqrt{-(a-1)(2 t a+1)\left(4 t^{2} a^{2}+2 t a^{2}-2 t a-a+1\right)}}{2 t a(2 t a+1)} \\
& =O(1) .
\end{aligned}
$$

As was the case in Model 17, we have chosen this combination of $x, \bar{x}, \xi_{ \pm}$so that the expression (115) is a power series in $t$. The coefficient $C_{1}$ is a simple rational function of $a, t$, while the other coefficients are algebraic. Note also that $C_{0}+C_{3}=0$. Using this partial fraction form we can compute the constant term as

$$
\left[x^{0}\right]\left\{\left(C_{0}+\frac{C_{1}}{x}+\frac{C_{2}}{1-x / \xi_{+}}+\frac{C_{3}}{1-\bar{x} \xi_{-}}\right) L(\bar{x})\right\}=C_{2} \cdot L\left(1 / \xi_{+}\right) .
$$

Thankfully we can express $L\left(1 / \xi_{+}\right)$in terms of $L(0)$. Return to equation (108), and notice that we can eliminate $L(y)$ by setting $x=\frac{t a(1+y)}{a-1}$. We can eliminate the kernel by setting $y=Y^{\prime}(t ; a)$ being the power series solution of $K\left(\frac{t a(1+y)}{a-1}, y\right)=0$ :

$$
Y^{\prime}(t ; a)=a-1-\frac{2 t^{2} a^{2}-\sqrt{-(a-1)(2 t a+1)\left(4 t^{2} a^{2}+2 t a^{2}-2 t a-a+1\right)}}{2 t a(t a+a-1)} .
$$

The combination

$$
\frac{\operatorname{ta}\left(1+Y^{\prime}\right)}{a-1}=1 / \xi_{+}
$$


hence we are left with an equation linking $L(1 / \xi+)$ with $L(0)$ :

$$
L\left(1 / \xi_{+}\right)=\frac{Y^{\prime}(a-1)}{a(a t+a-1)\left(a t Y^{\prime}+a t-a Y^{\prime}+Y^{\prime}\right)}\left(1-(a-1)^{2} L(0)\right) .
$$

So putting together all the parts from the constant term we get the equation

$$
\begin{aligned}
L(0)+C_{2} \cdot \frac{Y^{\prime}(a-1)}{a(a t+a-1)\left(a t Y^{\prime}+a t-a Y^{\prime}+Y^{\prime}\right)}\left(1-(a-1)^{2} L(0)\right)= \\
\left(1-L(0)(a-1)^{2}\right) \cdot\left[x^{0}\right]\{H(x, Y(x) ; a)\} .
\end{aligned}
$$

Isolating $L(0)$ gives

$$
\begin{aligned}
L(0)= & \frac{1}{(a-1)^{2}} \\
& \quad-\frac{1}{(a-1)^{2}\left(1-\frac{C_{2} \cdot Y^{\prime}(a-1)}{a(a t+a-1)\left(a t Y^{\prime}+a t-a Y^{\prime}+Y^{\prime}\right)}-(a-1)^{2} \cdot\left[x^{0}\right]\{H(x, Y(x) ; a)\}\right)} .
\end{aligned}
$$

Hence we have shown that $L(0)=Q(0,0)=G(t ; a, a)$ can be written as (essentially) the reciprocal of a D-finite function. Therefore it is, at worst, D-algebraic.

\section{Model 19 - Interacting Kreweras walks}

We have so far been unable to solve this model for general $(a, b)$; however, see the end of this section for some other recent results. Here, we solve it along the line $(a, a)$. Our solution follows, essentially, Bousquet-Mélou's algebraic kernel method solution [8] but with additional complications. The functional equation when $b=a$ and kernel are:

$$
\begin{aligned}
a^{2} x y K \cdot Q(x, y) & =1+(a x-x-a t) a y L(y)+(a y-y-a t) a x L(x)-(a-1)^{2} x y L(0) \\
\text { and } K & =1-t(\bar{x}+\bar{y}+x y)
\end{aligned}
$$

where we have written $L(x)=Q(x, 0)=Q(0, x)$.

Following the method in [8], we use the kernel symmetries $(x, y) \mapsto(\overline{x y}, y),(x, \overline{x y})$ to generate two new equations. A little linear algebra allows us to remove all of the $L(\overline{x y})$ terms and then we divide by the kernel:

$$
\begin{gathered}
(a x y t-a+1) Q(x, y)+(a t-a y+y) \bar{y} Q(x, \overline{x y})-(a t-a x+x) \bar{x} Q(\overline{x y}, y)= \\
\frac{a x^{2} y^{2} t-a x y+a x y-a y t+x y}{x y a^{2} K}-2 \frac{(a t-a y+y)(a x y t-a+1)}{a y K} \cdot L(x) \\
-\frac{(a-1)^{2}\left(a x^{2} y^{2} t-a x y+a x t-a y t+x y\right)}{x y a^{2} K} \cdot L(0) .
\end{gathered}
$$

This is quite a bit messier than the $(a, b)=(1,1)$ case, but we can still proceed in the same manner; our next step is to take the constant term with respect to $y$ of both sides. We do each side in turn. 


\subsection{Constant term of the left-hand side}

Consider each of the 3 terms in turn.

- The first term is easy to compute - the constant term is

$$
\left[y^{0}\right]\{(a x y t-a+1) Q(x, y)\}=(1-a) L(x) .
$$

- The second term is a little messier, but again gives just

$$
\left[y^{0}\right]\{(a t-a y+y) \bar{y} Q(x, \overline{x y})\}=(1-a) L(x) .
$$

- The third is a little more complicated and we write its constant term in terms of the diagonal of $Q$ :

$$
\left[y^{0}\right]\{(a x-a t-x) \bar{x} Q(\overline{x y}, y)\}=(a-1-a t \bar{x}) Q_{d}(\bar{x}),
$$

where $Q_{d}(x)=\left[q^{0}\right]\{Q(x q, \bar{q})\}$ is the diagonal of $Q(x, y)$.

So the constant term of the left-hand side is

$$
\left[y^{0}\right]\{\mathrm{LHS}\}=2(1-a) L(x)+(a-1-a t \bar{x}) Q_{d}(\bar{x}) .
$$

\subsection{The constant term of the right-hand side}

The constant term of this side of the equation is more involved. We first note that the right-hand side can be written as

$$
\mathrm{RHS}=C_{1}+\frac{C_{2}}{K},
$$

where both $C_{1}, C_{2}$ are independent of $y$ :

$$
\begin{aligned}
& C_{1}=\frac{(a-1)^{2}}{a} L(0)+2(1-a) L(x)-\frac{1}{a} ; \\
& C_{2}=\frac{(a-1)^{2}(2 t a-x) L(0)}{x a^{2}}-2 \frac{\left(a^{2} t^{2} x^{2}+a^{2} t-t a-x a+x\right) L(x)}{x a}-\frac{2 t a-x}{x a^{2}} .
\end{aligned}
$$

The constant term of $C_{1}$ is straightforward - it is just $C_{1}$, but the constant term of $C_{2} / K$ requires some work.

It suffices to consider the constant term $\left[y^{0}\right]\left\{\frac{1}{K}\right\}$. As for Model 17 we compute a partial fraction decomposition

$$
\frac{1}{K}=A_{1}+\frac{A_{2}}{1-y / \gamma_{+}}+\frac{A_{3}}{1-\bar{y} \gamma_{-}}
$$


where $\gamma_{ \pm}$are $y$-solutions of the kernel:

$$
\begin{aligned}
& \gamma_{+}=\frac{x-t+\sqrt{(x-t)^{2}-4 t^{2} x^{3}}}{2 t x^{2}}=\bar{x} t^{-1}+O(1) ; \\
& \gamma_{-}=\frac{x-t-\sqrt{(x-t)^{2}-4 t^{2} x^{3}}}{2 t x^{2}}=t+O\left(t^{2}\right) .
\end{aligned}
$$

The constant term with respect to $y$ is $A_{1}+A_{2}+A_{3}$ and simplifies to

$$
\left[y^{0}\right]\left\{\frac{1}{K}\right\}=A_{1}+A_{2}+A_{3}=\frac{1}{\sqrt{\Delta}}, \quad \text { where } \Delta=(1-t \bar{x})^{2}-4 x t^{2} .
$$

So finally, the constant term of the right-hand side is just

$$
\left[y^{0}\right]\{\mathrm{RHS}\}=C_{1}+\frac{C_{2}}{\sqrt{\Delta}} .
$$

\subsection{Reassembly and another constant term}

Equating the constant terms of the two sides and cleaning up gives us

$$
(a-1-a t \bar{x}) Q_{d}(\bar{x})-\frac{(a-1)^{2}}{a} L(0)+\frac{1}{a}=\frac{C_{2}}{\sqrt{\Delta}} .
$$

Notice that on the left-hand side of the above equation we only have non-positive powers of $x$. We can further separate the powers of $x$ by careful factorisation of $\Delta$ into three series - again following [8]

$$
\Delta=(1-t \bar{x})^{2}-4 x t^{3}=\left(1-x \delta_{1}\right)\left(1-\bar{x} \delta_{2}\right)\left(1-\bar{x} \delta_{3}\right),
$$

where the roots $\delta_{i}$ are readily computed:

$$
\begin{aligned}
& \delta_{1}=\frac{1}{4 t^{2}}-2 t-12 t^{4}+\cdots ; \\
& \delta_{2}=t+2 t^{5 / 2}+6 t^{4}+\cdots ; \\
& \delta_{3}=t-2 t^{5 / 2}+6 t^{4}-\cdots
\end{aligned}
$$

(In [11] these are referred to as the branch points of $\Delta$.) Then write

$$
\begin{aligned}
\Delta_{+} & =\left(1-x / \delta_{1}\right)=1-4 x t^{2}-32 x t^{5}+\cdots, \\
\Delta_{-} & =\left(1-\bar{x} \delta_{2}\right)\left(1-\bar{x} \delta_{3}\right)=1-2 \bar{x} t+\bar{x}^{2} t^{2}+\cdots \\
\text { and } \Delta_{0} & =4 t^{2} \delta_{1}=1-8 t^{3}-48 t^{6}+\cdots .
\end{aligned}
$$

By multiplying through $\Delta_{-}$we almost separate the equation into terms that contain strictly negative powers of $x$ and terms that contain strictly positive powers of $x$ :

$$
\begin{aligned}
& \sqrt{\Delta_{-}}\left(\frac{(a-1)^{2}}{a} L(0)+(t a-x a+x) \bar{x} Q_{d}(\bar{x})+\frac{1}{a}\right)= \\
& \frac{1}{\sqrt{\Delta_{0} \Delta_{+}}}\left(\frac{(a-1)^{2}(2 t a-x) L(0)}{x a^{2}}-2 \frac{\left(a^{2} t^{2} x^{2}+a^{2} t-t a-x a+x\right) L(x)}{x a}-\frac{2 t a-x}{x a^{2}}\right) .
\end{aligned}
$$


Notice that the left-hand side now contains only powers of $x^{0}, x^{-1}, x^{-2}, \ldots$ while the righthand side contains only powers of $x^{-1}, x^{0}, x^{1}, x^{2}, \ldots$ We can complete the separation of powers by moving the coefficient of $x^{0}$ from the left-hand side to the right, and moving the coefficient of $x^{-1}$ from the right-hand side to the left.

The constant term of the left-hand side is

$$
\left[x^{0}\right]\{\text { LHS }\}=\frac{1}{a}+\frac{a-1}{a} L(0)
$$

and the coefficient of $x^{-1}$ on the right-hand side is

$$
\left[x^{-1}\right]\{\operatorname{RHS}\}=\frac{2 t((a-1) L(0)+1)}{\sqrt{\Delta_{0}}} .
$$

Moving these terms to either side we can completely separate the equation into strictly negative powers of $x$ on the left-hand side, and non-negative powers of $x$ on the right-hand side. This then gives two new equations (obtained by extracting non-negative powers of $x$ and strictly negative powers of $x$ ):

$$
\begin{aligned}
& \sqrt{\Delta_{-}}\left(\frac{(a-1)^{2}}{a} L(0)+(t a-x a+x) \bar{x} Q_{d}(\bar{x})+\frac{1}{a}\right)-\frac{2 t((a-1) L(0)-1)}{x \sqrt{\Delta_{0}}}=0 ; \quad \\
& \frac{1}{a}+\frac{a-1}{a} L(0)-\frac{2 t((a-1) L(0)-1)}{x \sqrt{\Delta_{0}}} \\
& -\frac{1}{\sqrt{\Delta_{0} \Delta_{+}}}\left(\frac{(a-1)^{2}(2 t a-x) L(0)}{x a^{2}}-2 \frac{\left(a^{2} t^{2} x^{2}+a^{2} t-t a-x a+x\right) L(x)}{x a}-\frac{2 t a-x}{x a^{2}}\right) \\
& =0 .
\end{aligned}
$$

The second of these is readily solved using the single-variable (ie. "classical") kernel method. Notice the coefficient of $L(x)$ is a polynomial in $x$. It has two $x$-solutions:

$$
\begin{aligned}
& \xi_{+}=\frac{a-1+\sqrt{(a-1)\left(1-a+4 a^{3} t^{3}\right)}}{2 a^{2} t^{2}}=\frac{a-1}{a^{2}} t^{-2}+O(t) ; \\
& \xi_{-}=\frac{a-1-\sqrt{(a-1)\left(1-a+4 a^{3} t^{3}\right)}}{2 a^{2} t^{2}}=a t+O\left(t^{4}\right) .
\end{aligned}
$$

Setting $x=\xi_{-}$in equation (151) leaves an equation in only $L(0)$ :

$$
(1-a) L(0)=\frac{a\left(2 a t-\xi_{-}\right)}{(2 a t-\xi)(1-a)+\left(\xi_{-} \sqrt{\Delta_{0}}-2 t\right) a \sqrt{\Delta_{+}\left(\xi_{-}\right)}},
$$

where $\Delta_{+}\left(\xi_{-}\right)$denotes substituting $x \mapsto \xi_{-}$in $\Delta_{+}$. Hence $L(0)=Q(0,0)$ is an algebraic function of $t$. In fact, it satisfies a degree 6 polynomial. 
An anonymous referee pointed out to us that it should be possible to use the methods of [11] to prove that $Q(x, 0)$ is not algebraic for arbitrary $a, b$. We agree but for now have not pursued this further.

We also point to two other current works by two of us (NRB and ALO) and a collaborator [3, 4]. In [4] we have obtained the solution for Model 20 (reverse Kreweras walks) for arbitrary $a, b$ (in fact, in greater generality - weight $c$ is associated with visits to the corner $(0,0)$ instead of weight $a b)$. The solution is algebraic for all $a, b, c$. This solution can then be used to solve Model 19 in full generality (the generating functions are identical for walks ending at the corner, but not for walks ending anywhere else), where for arbitrary $a, b, c$ and arbitrary endpoint, the solution is D-finite (but probably not algebraic). Note that this is compatible with the referee's comment above. The conference paper [3] is an abridged version of [4].

\section{Discussion}

The results we have proved are summarised in Theorems 1 to 5. We now summarise our unproven observations from Tables 1 through 6 as three conjectures and one question.

While have found closed form expressions for $G(t ; a, b)$ for several models as the ratio or reciprocal of D-finite functions - suggesting a D-algebraic solution - we are unable to prove that these are, in fact, not D-finite. Additionally, we have not been able to guess D-finite solutions for these models based on analysis of long series. This leads to the following conjecture.

Conjecture 1. The generating functions $G(t ; a, a)$ and $G(t ; a, b)$ for Models 1, 3, 4, 17, 18 and 22 are not D-finite.

We were able to find D-finite solutions for models possessing a symmetry across a vertical line, but only when $b=1$. We have been unable to find or guess solutions for any other values of $a$ and $b$. On the basis of this we conjecture the following:

Conjecture 2. The generating functions $G(t ; a, b)$ for Models 5-16 are not D-finite except when $b=1$.

The generating function $G(t ; a, b)$ is equal in Models 19 and 20 and we have shown that it is algebraic when $a=b$. We have not solved the model at more general values of $a$ and $b$, however we have managed to guess algebraic solutions at various integer values of $a$ and $b$ and based on this we conjecture:

Conjecture 3. The generating functions $G(t ; a, b)$ for Models 19 and 20, Kreweras and reverse Kreweras walks, are algebraic for all $a, b$.

As mentioned in the previous section, this models have also been studied in $[3,4]$, where Conjecture 3 appears to have been confirmed.

We have had far less success with Models 21 and 23 and have not been able to solve them at any non-trivial values of $a$ and $b$. We were able to guess D-finite solutions to 
Model 21 at $a=1$ or $b=1$, but we have not been able to show that those solutions are transcendental. We have not been able to guess algebraic solutions at those values. For Model 23 we were able to guess an algebraic solution when $b=1$ and a D-finite solution at $a=1$. Similar to Model 21, we have not been able to prove transcendence of the D-finite solution at $a=1$, nor have we been able to guess an algebraic solution there. Based on this we ask the question:

Question 4. Are the generating functions $G(t ; a, 1), G(t ; 1, b)$ for Model 21, double Kreweras walks, and $G(t ; 1, b)$ for Model 23, Gessel walks, D-finite and not algebraic?

If so, then the generating functions $G(t ; a, b)$ for these models are not algebraic in contrast with $G(t ; 1,1)$.

Some comments regarding solvability and symmetry are worthwhile. Introducing different weights, $a$ and $b$, on the boundary breaks the diagonal symmetry of the quarter plane. Models that are both horizontally and vertically symmetric (ie. Models $1-4$ ), remain soluble even though the analytic nature of the generating function changes - see Conjecture 1. The other problem that behaves in the same way is Model 17, which possesses a symmetry along the line $y=-x$. Breaking symmetry along the line $y=x$ does not seem to change its solubility.

On the other hand, Models 5-16 only have symmetry around a vertical line and we have only been able to solve these problems when there is no weight present on the vertical boundary. As soon as the vertical boundary is weighted, the broken symmetry renders the problem more difficult and we have been unable to either solve or guess the generating function - see Conjecture 2.

We have been unable to solve Models 18, 19, 20 and 21 when $a \neq b$. These four models possess symmetry along the line $y=x$ (but not horizontal or vertical symmetry), and unsurprisingly our solution method, which relies on this symmetry, fails in these cases. In spite of this, we have strong numerical evidence that $G(t ; a, b)$ is algebraic for Models 19 and 20 - see Conjecture 3 . However, see also [3, 4].

Models 1 and 22 can be solved by another method - first solve the $a=1$ and $b=1$ cases separately (with solutions given as constant terms of algebraic functions), and then substitute these into the general functional equation. This gives the general generating function as a rational expression involving the $a=1$ and $b=1$ solutions. Unfortunately we have been unable derive analogous solutions for any other models.

We next make a few comments about the impediments to using the variations of the kernel method to the problems above. Arguably, these difficulties fall into three categories. First, and perhaps most prosaic, is that when we introduce boundary weights, the coefficients of equations become more complicated. While, in principle, this does not prevent a solution, in practice it does. For example, we believe that Model 21 might be soluble via a similar approach to that used for Models 19 and 20, however the required manipulations quickly become byzantine.

Perhaps more substantive is that for a great number of the models we have not solved - including all of 5-16 - a key coefficient is no longer rational. More specifically, if we attempt to replicate the approach in Section 3, the coefficient of $Q(\bar{x}, 0)$ in the equivalent 
of equation (35) is algebraic and not rational. Because of this, we are not able to express the required constant term as a simple substitution. Indeed, it is not clear that a simple closed form for the required constant term exists.

Finally, we have attempted to use the so-called full orbit sum kernel method, in which one sums over all the symmetries of the kernel in order eliminate boundary terms - see Proposition 8 from [9]. Unfortunately, it does not appear to be possible to eliminate sufficient boundary terms. For example, in Model 5, a sum over the 4 kernel symmetries allows one to remove most, but not all, of the boundary terms. For general $a, b$, one is left with an equation of the form

$$
[\text { bulk terms }] K=[\text { coefficient }] Q(0,0)+[\text { coefficient }] Q(x, 0)
$$

and it does not appear that any improvement is possible. It may be possible to follow the methods used to solve Kreweras walks (see Section 8 and $[3,4,8]$ ) to further process this equation towards a solution.

It is certainly worth investigating whether other methods, such as those presented in [11], recent differential Galois theory techniques [10], contour integral expressions [11, 25], or the Tutte invariant method [5], can be adapted to handle these interacting boundary problems.

\section{Acknowledgements}

Financial support from the Australian Research Council via its Discovery schemes (DE170100186 and DP160103562) is gratefully acknowledged by N. R. Beaton and A. L. Owczarek respectively. A. Rechnitzer acknowledges support from NSERC Canada via a Discovery Project Grant. N. R. Beaton and A. Rechnitzer also received support from the PIMS Collaborative Research Group in Applied Combinatorics. The authors thank an anonymous referee for helpful comments.

\section{References}

[1] M. Albert and M. Bousquet-Mélou. Permutations sortable by two stacks in parallel and quarter plane walks. Eur. J. Combin., 43:131-164, 2015.

[2] M. N. Barber and B. W. Ninham. Random and Restricted Walks: Theory and Applications. CRC Press, 1970.

[3] N. R. Beaton, A. L. Owczarek, and R. Xu. Quarter-plane lattice paths with interacting boundaries: Kreweras and friends (extended abstract for FPSAC'19). In Séminaire Lotharingien de Combinatoire, 2019. To appear.

[4] N. R. Beaton, A. L. Owczarek, and R. Xu. Quarter-plane lattice paths with interacting boundaries: the Kreweras and reverse Kreweras models. Preprint, arXiv: 1905. 10908, 2019. 
[5] O. Bernardi, M. Bousquet-Mélou, and K. Raschel. Counting quadrant walks via Tutte's invariant method (extended abstract for FPSAC'16). In Discrete Math. Theor. Comput. Sci. Proc., 2016.

[6] A. Bostan and M. Kauers. Automatic classification of restricted lattice walks (extended abstract for FPSAC'09). In Discrete Math. Theor. Comput. Sci. Proc., pages 201-215, 2009.

[7] M. Bousquet-Mélou. Counting walks in the quarter plane. In Mathematics and Computer Science II, pages 49-67. Springer, 2002.

[8] M. Bousquet-Mélou. Walks in the quarter plane: Kreweras' algebraic model. Ann. Appl. Prob., 15(2):1451-1491, 2005.

[9] M. Bousquet-Mélou and M. Mishna. Walks with small steps in the quarter plane. In Algorithmic Probability and Combinatorics, volume 520 of Contemporary Mathematics, pages 1-40. 2010.

[10] T. Dreyfus, C. Hardouin, J. Roques, and M. F. Singer. On the nature of the generating series of walks in the quarter plane. Invent. Math., pages 1-65, 2018.

[11] G. Fayolle, R. Iasnogorodski, and V.A. Malyshev. Random Walks in the QuarterPlane. Springer, 1999.

[12] P. Flajolet and R. Sedgewick. Analytic Combinatorics. Cambridge University Press, 2009.

[13] I. Gessel. A probabilistic method for lattice path enumeration. J. Statist. Plann. Inference, 14(1):49-58, 1986.

[14] I. Gessel, J. Weinstein, and H. S. Wilf. Lattice walks in $\mathbb{Z}^{d}$ and permutations with no long ascending subsequences. Electron. J. Combin, 5(1):\#R2, 1998.

[15] I. Gessel and D. Zeilberger. Random walk in a Weyl chamber. Proc. Amer. Math. Soc., 115(1):27-31, 1992.

[16] E.J. Janse van Rensburg. The Statistical Mechanics of Interacting Walks, Polygons, Animals and Vesicles. OUP Oxford, 2nd edition, 2015.

[17] M. Kauers, M. Jaroschek, and F. Johansson. Ore polynomials in Sage. In Computer Algebra and Polynomials, pages 105-125. Springer, 2015.

[18] M. Kauers, C. Koutschan, and D. Zeilberger. Proof of Ira Gessel's lattice path conjecture. Proc. Nat. Acad. Sci., pages pnas-0901678106, 2009.

[19] M. Kauers and R. Yatchak. Walks in the quarter plane with multiple steps (extended abstract for FPSAC'15). In Discrete Math. Theor. Comput. Sci. Proc., pages 25-36, 2015.

[20] G. Kreweras. Sur une classe de problèmes de dénombrement liés au treillis des partitions des entiers. Cahiers du Bureau universitaire de recherche opérationnelle Série Recherche, 6:9-107, 1965.

[21] L. Lipshitz. The diagonal of a D-finite power series is D-finite. J. Algebra, 113(2):373378, 1988. 
[22] L. Lipshitz. D-finite power series. J. Algebra, 122(2):353-373, 1989.

[23] M. Mishna. Classifying lattice walks restricted to the quarter plane. J. Combin. Theory Ser. A, 116(2):460-477, 2009.

[24] G. Pólya. Über eine aufgabe der wahrscheinlichkeitsrechnung betreffend die irrfahrt im straßennetz. Mathematische Annalen, 84(1-2):149-160, 1921.

[25] K. Raschel. Counting walks in a quadrant: a unified approach via boundary value problems. J. Europ. Math. Soc., 14(3):749-777, 2012.

[26] F. Spitzer. Principles of Random Walk, volume 34. Springer Science \& Business Media, 2013.

[27] R. P. Stanley. Enumerative Combinatorics, Volume 2. Cambridge University Press, Cambridge, 2001.

[28] R. Tabbara, A. L. Owczarek, and A. Rechnitzer. An exact solution of two friendly interacting directed walks near a sticky wall. J. Phys. A: Math. Theor., 47(1):015202, 2013.

[29] R. Tabbara, A. L. Owczarek, and A. Rechnitzer. An exact solution of three interacting friendly walks in the bulk. J. Phys. A: Math. Theor., 49(15):154004, 2016.

[30] The Sage Developers. SageMath, the Sage Mathematics Software System (Version 8.2), 2018. http://www. sagemath.org. 